DOI: 10.1002/ ((please add manuscript number))

Article type: Full Paper

\title{
Signatures of Quantized Energy States in Solution-Processed Ultra-Thin Layers of Metal Oxide Semiconductors and Their Devices
}

John G. Labram ", Yen-Hung Lin, Kui Zhao, Ruipeng Li, Stuart R. Thomas, James Semple, Maria Androulidaki, Lamprini Sygellou, Martyn McLachlan, Emmanuel Stratakis, Aram Amassian, Thomas D. Anthopoulos ${ }^{*}$

Dr. J. G. Labram, Mr Y.-H. Lin, Dr. S. R. Thomas, Mr. J. Semple, Prof. T. D. Anthopoulos Department of Physics

Blackett Laboratory

Imperial College London

London, SW7 2AZ, United Kingdom

E-mail: t.anthopoulos@imperial.ac.uk,j.labram@imperial.ac.uk

Mr Y.-H. Lin

Dutch Polymer Institute (DPI)

P.O. Box 902, 5600 AX Eindhoven

The Netherlands

Miss. M. Androulidaki, Dr. L. Sygellou, Dr. Emmanuel Stratakis

Foundation for Research and Technology Hellas (FORTH)

Institute of Electronic Structure and Lasers (IESL)

Heraklion Crete and Institute of Chemical Engineering Sciences (ICEHT),

Patras, Greece

Dr. M. McLachlan

Department of Materials, Royal School of Mines

Imperial College London, London, SW7 2AZ, United Kingdom

Dr. K. Zhao, Prof. A. Amassian

Materials Science and Engineering, Division of Physical Sciences and Engineering

King Abdullah University of Science and Technology, Thuwal 23955-6900, Saudi Arabia

Dr. R. Li,

Cornell High Energy Synchrotron Source

Cornell University, Ithaca, NY, 14850, U.S.A.

Keywords: Solution-Processed Semiconductors, Semiconducting Oxides, Energy Quantization, Resonant Tunneling Diodes, Zinc Oxide 


\title{
WILEY-VCH
}

\begin{abstract}
Physical phenomena such as energy quantization have to-date been overlooked in solutionprocessed inorganic semiconducting layers, owing to heterogeneity in layer thickness uniformity unlike some of their vacuum-deposited counterparts. Recent reports of the growth of uniform, ultra-thin $(<5 \mathrm{~nm})$ metal-oxide semiconductors from solution, however, have potentially opened the door to such phenomena manifesting themselves. Here, we develop a theoretical framework for energy quantization in inorganic semiconductor layers with appreciable surface roughness, as compared to the mean layer thickness, and present experimental evidence of the existence of quantized energy states in spin-cast layers of zinc oxide $(\mathrm{ZnO})$. As-grown $\mathrm{ZnO}$ layers are found to be remarkably continuous and uniform with controllable thicknesses in the range $2-20 \mathrm{~nm}$ and exhibit a characteristic widening of the energy band gap with reducing thickness in agreement with theoretical predictions. Using sequentially spin-casted layers of $\mathrm{ZnO}$ as the bulk semiconductor and quantum well materials, and gallium oxide or organic self-assembled monolayers as the barrier materials, we demonstrate two terminal electronic devices the current-voltage characteristics of which resemble closely those of double-barrier resonant-tunneling diodes. As-fabricated alloxide/hybrid devices exhibit a characteristic negative-differential conductance region with peak-to-valley ratios in the range $2-7$.
\end{abstract}

\section{Introduction}

Solution-processed metal-oxide semiconductors ${ }^{1,2}$ offer great promise for low-cost, largearea electronics and are currently the focus of great academic and commercial interest. ${ }^{3}$ In the past few years significant progress has been made in reducing the processing temperature of high-performance solution processable metal-oxide semiconductors and devices, ${ }^{4}, 5$ 


\section{WILEY-VCH}

traditionally a significant technological hurdle for oxide semiconductors. Furthermore, the growth of highly uniform and ultra-thin $(<5 \mathrm{~nm})$ films of polycrystalline zinc oxide $(\mathrm{ZnO})$ has also been demonstrated from solution at low temperatures $\left(180^{\circ} \mathrm{C}\right)$ leading to the realization of thin-film transistors (TFTs) with field-effect mobilities in excess of $10 \mathrm{~cm}^{2} / \mathrm{Vs}^{6}{ }^{6}$ Whilst the effect of channel thickness has recently been considered in solution-processed metal oxide transistors, ${ }^{7}$ work has thus far focused upon the impact of the channel dimensions on the electrical stability of the devices rather than on the possibility of forming quantized carrier states. Traditional semiconductor systems such as GaAs/AlGaAs deposited via sophisticated growth techniques, such as chemical vapor deposition (CVD) or molecular-beam epitaxy (MBE), are known to exhibit quantized sub-bands when carriers are confined in potential wells of dimensions below 5-10 $\mathrm{nm} .{ }^{8}$ Unlike traditional inorganic systems, however, solutiondeposited oxide semiconducting layers are characterized by rough surfaces with root-meansquare (RMS) roughness often on the order of several nm. ${ }^{9}$ This characteristic combined with the amorphous / polycrystalline nature of most semiconducting oxides, has generally led to this family of materials being overlooked for use in low-dimensional devices. However, regardless of whether the system is smooth or rough or whether the transport of carriers in the semiconducting layer is described by a hopping-like or a band-like mechanism, if carriers are spatially confined in one dimension, the Schrödinger equation dictates that the energies available to these carriers will be restricted to quantized energy levels rather than a continuum. ${ }^{10}$ Therefore, ultra-thin metal oxide semiconducting layers grown from solution at low temperatures could potentially provide an ideal test-bed for studying the possible formation of quantized carrier states. 


\section{WILEY-VCH}

In this work, we study the formation of quantized energy levels in ultra-thin layers of $\mathrm{ZnO}$ processed from solution at $200{ }^{\circ} \mathrm{C}$ in ambient air. By tuning the molarity of the aqueous precursor solution and the spin-speed during deposition, growth of conformal, polycrystalline $\mathrm{ZnO}$ layers with controllable thicknesses ranging between 2-20 $\mathrm{nm}$ is demonstrated. By means of optical absorption and photo-luminescent spectroscopy, we studied the impact of layer thickness on the optical properties of $\mathrm{ZnO}$. Obtained results revealed a characteristic widening of the semiconductor bandgap in agreement with theoretical predictions for energy level quantization below a critical layer thickness. Further supporting evidence for the existence of quantized energy states in ultra-thin $\mathrm{ZnO}$ layers, were obtained via electrical characterization of quantum-well structures based on all-oxide as well as on metal oxide/organic hybrid materials combinations.

\section{Deposition of Ultra-Thin ZnO Layers from Solution}

Recently $^{6,11}$ we demonstrated the ability to deposit ultra-thin, highly-uniform films of semiconducting $\mathrm{ZnO}$ by spin-casting from an aqueous $\mathrm{ZnO}$ precursor solution followed by a thermal annealing step at $\sim 180{ }^{\circ} \mathrm{C}$. By employing various precursor concentrations and spinning speeds, the process has been employed here to produce $\mathrm{ZnO}$ films with mean thicknesses ranging from $\sim 2 \mathrm{~nm}$ to $\sim 24 \mathrm{~nm}$. In Figure 1(a) we show a high-resolution transmission electron microscope (HRTEM) cross-sectional image of a solution-processed $\mathrm{ZnO}$ film on $\mathrm{SiO}_{2}$ substrate at two different magnifications. The film is found to be visibly polycrystalline with a mean thickness of approximately $6.5 \mathrm{~nm}$. In Figure 1(b) we show a grazing incident $\mathrm{x}$-ray diffraction (GID) spectrum of a $\mathrm{ZnO}$ layer processed under identical conditions, again indicating a randomly oriented polycrystalline structure. 


\section{WILEY-VCH}

Tapping-mode atomic force microscopy (AFM) images of a similar film deposited onto quartz are displayed in Figure 1(c)-(f). In the large scan images of Figure 1(c)-(d), clear straight lines-like features are observed. These are attributed to the drying of the precursor solution in the presence of centrifugal forces during spin-casting. Although the film is generally continuous, small voids can be observed in the highest magnification images of Figures 1(e)-(f), which are believed to show the surface of the quartz substrate. By assuming that the AFM tip makes physical contact with the substrate surface at least once during the full scan, one can extract the height-profile of the $\mathrm{ZnO}$ layer directly from these AFM images and accurately assess the degree of substrate coverage. In Figure 1(g) we show the experimental film thickness height distribution extracted from the AFM image of Figure 1(e), fitted to a Gaussian distribution with a mean thickness of $6.8 \mathrm{~nm}$ and standard deviation of 0.9 nm. This is in good agreement with the HRTEM image of Figure 1(a) as well as further HRTEM images taken for various $\mathrm{ZnO}$ layers with different thicknesses. Field-effect transistor measurements carried out on $\mathrm{ZnO}$ films grown using identical experimental conditions yielded good semiconducting properties, further confirming the good substrate coverage and continuity of these layers (see Supporting Information Section S1).

\section{Energy Level Quantization in Rough Semiconducting Films}

Energy quantization in traditional semiconductor systems such as GaAs / AlGaAs has previously been studied via optical absorption measurements. ${ }^{8}$ In their systems, Dingle et.al. observed an optical blue shift in the absorption spectra as the semiconductor thickness was reduced. ${ }^{8}$ Due to the very high conduction band energy of quartz, a thin layer of $\mathrm{ZnO}$ of thickness $L$, on quartz, can here be approximated by an infinite quantum well. Finite quantum well energies were also calculated using known techniques ${ }^{12}$ (data not shown), but the results 


\section{WILEY-VCH}

were found to be negligibly different from those evaluated using the below infinite quantumwell approximation. If we define the dimension perpendicular to the substrate surface as $z$, then the energy of conduction band states available to electrons confined to an infinite quantum well can be described using: ${ }^{13}$

$$
E_{n}=E_{x y}+\frac{n^{2} h^{2}}{8 m^{*} L^{2}}
$$

Here, $E_{x y}$ is the energy associated with the electron in the (unconfined) $x y$-plane, $n$ is a positive integer, $h$ is the Planck Constant, $m^{*}$ is the effective mass of electrons in the semiconductor and $L$ is the thickness of the quantum well in the $z$-direction. As $L$ is reduced the energy of the first electron state $(n=1)$ and hence the conduction band minimum (CBM), increases. The energy of the first allowed transition from valance band to the conduction band similarly increases, resulting in a blue-shift in the optical absorption spectrum of the film. ${ }^{8}$

For semiconductors deposited via molecular-beam epitaxy (MBE) or chemical vapor deposition (CVD), the layer uniformity is extremely high and each incident photon can be assumed to encounter a single well width $(L)$. However, since the roughness of solutionprocessed semiconductor layers considered here is expected to be non-negligible, it is here more appropriate to describe each incident photon as encountering a well of width $L$ with a probability $P\left(\mu_{L}, \sigma_{L}\right)$, where $\mu_{L}$ is the mean well width and $\sigma_{L}$ is the well width standard deviation. The thickness probability distribution $P\left(\mu_{L}, \sigma_{L}\right)$ is here approximated to be Gaussian (see Figure 1(g) for verification). In this case the probability of an incident photon encountering a well of thickness $L$ can be described by: 


$$
P\left(L, \mu_{L}, \sigma_{L}\right)=\frac{1}{\sigma_{L} \sqrt{2 \pi}} \exp \left[-\frac{\left(L-\mu_{L}\right)^{2}}{2 \sigma^{2}}\right]
$$

The second term in Eq. (1) is the energy of the quantized sub-band states in the conduction band. By substituting this into Eq. (2) above the probability of an incident photon encountering a well with sub-band energy levels $E_{n}$ can then be described by:

$$
P\left(E_{n}, \mu_{L}, \sigma_{L}\right)=\frac{1}{\sigma_{L} \sqrt{2 \pi}} \exp \left[\frac{1}{2 \sigma_{L}^{2}}\left(\frac{n h \mu_{L}}{\sqrt{2 m^{*} E_{n}}}-\frac{n^{2} h^{2}}{8 m^{*} E_{n}}-\mu_{L}^{2}\right)\right]
$$

For illustrative purposes this distribution is plotted in Figure 2 for the first four states of an infinite quantum well with $\mu_{L}=2 \mathrm{~nm}, \sigma_{L}=0.1 \mathrm{~nm}$ and $m^{*}=0.29 m_{e}$ (where $m_{e}$ is the electron rest-mass in a vacuum). Areas of dark green represent the energies with the highest probability for a given $n$, while areas of white the energies with probability zero. Hole states in the valence band are similarly expected to be quantized, however since the hole effective mass in $\mathrm{ZnO}$ is significantly higher than that of electrons ${ }^{14}$ these states have been neglected for the purposes of this study. Despite broadening, a range of energies are clearly forbidden (in particular close to the $n=1$ sub-band). This illustrates that it is indeed appropriate to consider quantized carrier states in somewhat rough or non-uniform semiconductor films if the mean thickness and its standard deviation, as indicated by the rms roughness are low enough.

It should be emphasized that in the above analysis, we are specifically interested in local height variations on the order of a few $\mathrm{nm}$, in contrast to large-scale non-uniformity over the 


\section{WILEY-VCH}

area of the substrate. A Gaussian distribution is chosen (aside from empirical reasons) because it implies that the roughness is homogeneous regardless of the area studied. By extracting surface roughness values from AFM scans over small areas (such as $1 \mu \mathrm{m} \times 1 \mu \mathrm{m}$ ) the values can be assumed to be representative of the length scales relevant to the phenomena described above.

\section{Optical Characterization of $\mathrm{ZnO}$ Layers}

To investigate the possible occurrence of energy quantization in our solution-processed oxide layers, the optical properties of several $\mathrm{ZnO}$ films of varying thickness were studied. Figure 3(a) shows the optical transmittance spectra of $4 \mathrm{ZnO}$ films of varying mean thickness deposited on quartz while Figure 3(b) shows the respective Tauc plots. ${ }^{15,16}$ All spectra were corrected for reflectance. As expected for quantized electron states, ${ }^{8}$ the onset of optical absorption is blue-shifted as the average layer thickness is reduced. This effect is better illustrated in Figure 3(c) where the change in optical band gap $\left(\Delta E_{G}\right)$-with respect to that of bulk $\mathrm{ZnO}-$ of $10 \mathrm{ZnO}$ films is plotted as a function of average film thickness (see Supporting Information Section S2). ZnO films with mean thickness $>20 \mathrm{~nm}$ showed a similar band gap of $\sim 3.25 \mathrm{eV}$. The latter value is approximated to be representative of bulk $\mathrm{ZnO}$ for the purposes of this analysis and is in good agreement with previously published results. ${ }^{17}$ The green band in Figure 3(c) illustrates the expected change in band gap, calculated using Eq. 2, with $n=1$. When evaluating $P\left(\mu_{L}, \sigma_{L}\right)$ in Figure 3(c) a standard deviation was employed that was $20 \%$ of the mean film thickness for all values. This is in agreement with what was observed experimentally for the $\mathrm{ZnO}$ films measured. The effective electron mass $\left(m^{*}\right)$ used was that of crystalline $\mathrm{ZnO}$, i.e. $m^{*}=0.29 m_{e}{ }^{18}$ 


\section{WILEY-VCH}

We note that the effective-mass approximation has been used to calculate the expected $\Delta E_{G}$ despite the fact that charge transport in solution-processed metal-oxide semiconductors is often described by a hopping-like transport mechanism rather than band-like transport. ${ }^{19} \mathrm{We}$ therefore expect that $m^{*}$ will not be constant as a function of energy. Despite this approximation a clear trend can be observed using this type of analysis, in line with an earlier (and only) report on vacuum-processed amorphous oxide superlattices. ${ }^{20}$ Previous studies have reported a blue-shift in bulk $\mathrm{ZnO}$ films deposited by CVD as the growth temperature is reduced, an effect attributed to changes in material crystallinity. ${ }^{21}$ The films studied here are known to be highly polycrystalline (see Figure $\mathbf{1}$ for example) for all layer thicknesses investigated. Similarly, phenomena such as the Burstein-Moss Effect can be ruled out, as even if the semiconductor films are degenerate (transistor measurements suggest they are not - see Supporting Information Section S1), the 3-dimensional electron density is unlikely to vary substantially as a function of film thickness.

Further evidence of energy quantization in these low-dimensional solution processed $\mathrm{ZnO}$ films was obtained by measuring the photoluminescence (PL) emission spectra of the $\mathrm{ZnO}$ as a function of layer thickness at $22 \mathrm{~K}$. Ultraviolet and visible emission bands are both observed in the spectra (Figure 3(d)). The former is attributed to exciton emission, while the latter to defects present in $\mathrm{ZnO}$, including oxygen vacancies, interstitial zinc and/or zinc vacancies. Analysis of the data reveals that the exciton peak is blue-shifted as the mean layer thickness of $\mathrm{ZnO}$ is reduced, as expected for quantized carrier states. ${ }^{22,23}$ The blue shift in the exciton peak is plotted as a function of mean film thickness in Figure 3(e). As it can be seen, the films with a mean thickness of $\sim 28.7 \mathrm{~nm}$ can be assumed to be representative of bulk $\mathrm{ZnO}$ since for quantized systems the exciton energy is known to scale as $\sim 1 / L^{2}$, where $L$ is the size 


\section{WILEY-VCH}

of the reduced dimension (i.e. layer thickness). ${ }^{24}$ This relationship has been fitted to the experimental data in Figure 1(e) with a prefactor of $2.5 \mathrm{meVnm}^{2}$. It can clearly be seen that this relation is in good agreement with the PL measurements (despite the technical challenges associated with the measurements), hence further supporting the existence of quantized states in these solution-deposited low-dimensional $\mathrm{ZnO}$ layers.

\section{Oxide-Based Double Barrier Resonant Tunneling Devices}

The existence of quantized energy states has in the past also been demonstrated through the observation of negative-differential conductance (NDC) in certain quantum well electronic

device structures. ${ }^{25-29}$ So-called double-barrier resonant tunneling diodes (RTDs) consist of a semiconducting quantum well confined between two ultra-thin potential barriers, in contact with bulk semiconducting layers on either side. The bulk semiconductors are in direct contact with metal contacts [i.e. the top and bottom Al contacts in Figure 4(a)] and act as electron "reservoirs". An idealized schematic of a ZnO-based RTD is shown in Figure 4(a) together with the corresponding energy band diagram in equilibrium at zero bias. The operating principle of an idealized RTD under biasing is illustrated in Figure 4(b)-(c). The quantized sub-bands confined in the center of the device act as a filter which allows the transmission of electrons at certain energies, whilst strongly attenuating those at other energies. When a bias $\left(V_{\text {Bias }}\right)$ is applied across the device the chemical potential of the bulk semiconductor region on the right of the diagram (the collector) is shifted down by $e V$ with respect to the chemical potential of the bulk semiconductor region on the left (the emitter). A linearly varying potential is then established across the device. When the quantized energy states (i.e. $n=1 \&$ 2) are resonant with the occupied electron states in the emitter, the probability of an electron tunneling from left to right is enhanced. As the applied voltage increases from zero, the device 


\section{WILEY-VCH}

current increases as the $n=1$ state becomes resonant with the emitter layer. However, as the forward bias increases further, the device current starts decreasing since the $n=1$ state moves below the conduction band minimum $(\mathrm{CBM})$ of the emitter. Experimentally this is manifest as a region of NDC in the current-voltage characteristics. ${ }^{25}$ This phenomenon has been observed in a range of material systems including: GaAs/AlGaAs, ${ }^{25,}{ }^{26} \mathrm{InAs} / \mathrm{AlSb},{ }^{27} \mathrm{Si} / \mathrm{SiGe},{ }^{28}$ graphene/BN, ${ }^{30} \mathrm{MgZnO} / \mathrm{ZnO},{ }^{31}$ and single molecules, ${ }^{32}$ but to-date never in a macroscopic device based on solution-processed materials.

Aside from the academic interest related to studying the properties of quantized states, RTDs have been proposed for a range of commercial applications. ${ }^{29}$ For example, due to their extremely fast response-time, ${ }^{26,27}$ RTDs have been envisaged as potential high-speed signal generators and detectors. ${ }^{29}$ Multiple-well resonant tunneling structures have also been considered as the foundations of multi-valued logic circuits. ${ }^{29,33}$ More complicated multiple quantum well structures have also been employed to create devices such as quantum-cascade lasers $^{34,35}$ and high-performance thermoelectric materials. ${ }^{36}$ Therefore, the ability to fabricate RTD-like devices employing cost-effective manufacturing techniques on large area substrates is scientifically appealing and potentially industry-relevant.

To investigate the possibility of realizing functional resonant tunneling-like devices from solution, we fabricated RTDs using solution deposited $\mathrm{ZnO}$ as the bulk semiconductor and QW layers, and $\mathrm{Ga}_{2} \mathrm{O}_{3}$ [Figure 4(a)] as the barrier layers material (see Experimental Section). $\mathrm{Ga}_{2} \mathrm{O}_{3}$ was chosen, firstly because it combines a large band gap $(\sim 4.9 \mathrm{eV})$ with appreciable $\mathrm{CBM}$ energy offset to $\mathrm{ZnO}$, and secondly because thin layers can be grown from solution at low temperatures. ${ }^{37}$ For the purpose of this study, the ionization potential (IP) and valence 


\section{WILEY-VCH}

band maxima (VBM) for both metal oxides were determined by ultraviolet photoelectron spectroscopy (UPS), respectively. The CBM energies for $\mathrm{ZnO}$ and $\mathrm{Ga}_{2} \mathrm{O}_{3}$ calculated were $\sim 3.8 \mathrm{eV}$ and $\sim 2.8 \mathrm{eV}$, respectively, in agreement with previously published data. The potential barrier height in the conduction band of the $\mathrm{Ga}_{2} \mathrm{O}_{3} / \mathrm{ZnO} / \mathrm{Ga}_{2} \mathrm{O}_{3}$ quantum well was therefore approximated to be $\sim 1.0 \mathrm{eV}$. Figure 4(d) shows the current-voltage (I-V) characteristics of a representative device measured repeatedly 5 times. Qualitative similar I-V curves were obtained for 100 s of devices processed in parallel. In each case electrical characterization was carried out at room temperature in ambient-pressure under nitrogen atmosphere. Despite the difference among the subsequent voltage sweeps, there is a clear region of NDC observable in this device. The variation in the voltage at which NDC occurs is likely to be due to charging/discharging effects -owed to the presence of electron traps- and/or material instability including ionic migration and/or polarization effects. ${ }^{38,}{ }^{39}$ Furthermore, significant operating hysteresis was observed between the forward and reverse bias sweeps (data not shown) making detailed analysis of the NDC voltage region very challenging.

In order to rule out other possible mechanisms that could give raise to NDC phenomena, we fabricated a series of control devices in parallel to the RTD devices shown in Figure 4(a). For each control device the deposition of the two $\mathrm{Ga}_{2} \mathrm{O}_{3}$ barrier layers was omitted during device fabrication, leading to the formation of the two-terminal $\mathrm{ZnO}$ device shown in Figure 5(a), with an approximate semiconductor thickness of $50 \mathrm{~nm}$. Out of over 40 control devices measured from the same batch, not a single I-V exhibited anything resembling NDC. A plot of representative I-V characteristics measured from 5 different control devices is shown in Figure 5(b). Similarly, control devices based on a single $\mathrm{Ga}_{2} \mathrm{O}_{3}$ barrier layer also failed to yield I-V characteristics with the NDC feature present. In parallel to the control devices, four 


\section{WILEY-VCH}

separate batches of RTDs [Figure 4(a)] were fabricated on different dates and electrically characterized using identical experimental conditions. Figures 5(c-f) display representative I$\mathrm{V}$ curves measured from different devices. On each occasion the two-terminal devices exhibited clear NDC regions occurring at comparable bias levels, with peak-to-valley current ratios in the range 2-7. The peak current-density recorded just before the NDC region was also found to vary with typical values in the range $5-50 \mathrm{~mA} / \mathrm{cm}^{2}$. This variation is most likely attributed to the difference in the effective active area between devices (that is the area of the device that exhibits resonant tunneling conduction) due to the non-uniform layer thickness of key device components such as the $\mathrm{ZnO}$ quantum well and the tunneling barrier (i.e. $\mathrm{Ga}_{2} \mathrm{O}_{3}$ ) width. Such non-uniformities could well lead to reduction of resonant tunneling events and hence to significant variation in the peak tunneling current between devices. Current work is currently focusing on mapping the resonant tunneling regions across the geometrical area of the RTDs using the current-AFM technique and will be reported in the future.

Despite the variation in the operating characteristics between different RTDs, asprepared devices could be stored for several days under ambient $\mathrm{N}_{2}$, after which the NDC feature remained measureable - a feature indicative of the good stability of these devices when not in operation. Despite theoretical predictions, however, the NDC feature was generally only observable in the first quadrant of the I-V plot and only in a few devices it appeared in the third quadrant. We attribute this to the fact that although conceptually the device structure in Figure 4(a) appears symmetric, in reality the bottom $\mathrm{Al} / \mathrm{ZnO}$ (bulk) interface and the top $\mathrm{ZnO}($ bulk $) / \mathrm{Al}$ interfaces are expected to be substantially different due to the specific fabrication steps followed (e.g. exposure of bottom Al electrode to sequential spin-casting of $\mathrm{ZnO}$ layers and thermal annealing steps during layer growth). This 


\section{WILEY-VCH}

characteristic "asymmetry" in the device structure is believed to be the most likely reason for the absence of NDC in the third quadrant of I-V characteristics.

Negative differential conductance has previously been observed in resistance random-access memory devices and was attributed to the migration of oxygen vacancies. ${ }^{40,41}$ Although we cannot rule out this as a possibility, given the absence of any NDC-like feature in our control devices in combination with the clear signatures of quantized energy states in thin $\mathrm{ZnO}$ films obtained via optical spectroscopy measurements (Figure 3) and the improved data sets that will be discussed in Section 6 for hybrid RTDs, we argue that the NDC feature presented in Figure 4(d) and Figures 5(c-f) being due to a resonant tunneling process is a more-likely explanation, and certainly a concept worthy of further investigation.

The maximum peak-to-valley current ratio measured from these devices was relatively high and on the order of $\sim 7$. The peak current before the region of NDC occurred at voltages in the range $0.6-1.1 \mathrm{~V}$. This peak current is expected to occur at a voltage approximately twice the energy of the peak electron transmittance divided by the electron charge $(e) .{ }^{25}$ By employing a basic model developed for traditional RTDs, ${ }^{42}$ the first peak transmittance in this device was approximated to occur at $0.12 \mathrm{eV}$. Therefore, NDC is expected to occur at around $0.24 \mathrm{~V}$ bias

- approximately 2 to 4 times lower than the experimentally measured voltages (see Supporting Information Section S3). These larger than expected voltages are likely to be due to applied potential being dropped elsewhere across the device structure. For example, a highresistance layer of $\mathrm{Al}_{2} \mathrm{O}_{3}$ is known to form between ultra-thin layers of $\mathrm{ZnO}$ and evaporated top Al electrodes after thermal annealing. ${ }^{6}$ The presence of such electrically insulating layer(s) among other parasitic effects, are therefore expected to introduce significant 


\section{WILEY-VCH}

deviations from the ideal device structure considered in the model. Furthermore, applying the model to a system formed between asymmetrically rough semiconductors/electrodes is also likely to introduce substantial differences in the predicated device operation.

\section{Hybrid Resonant Tunneling Devices}

In an attempt to improve the device performance and also simplify the manufacturing process, we fabricated $\mathrm{ZnO}$-based devices using insulating organic self-assembled monolayers (SAM) as the tunneling barrier materials. Two types of organic molecules were investigated, namely hexamethyldisilazane (HMDS) and phosphonohexadecanoic acid (PHDA) [Figure 6(a)]. Both SAM molecules are known to form dense and conformal self-assembled monolayers on solid surfaces with suitable chemistry ${ }^{43-46}$ and have been used extensively as surface passivation agents, ${ }^{47}$ surface energy modifiers ${ }^{45,48}$ and ultra-thin gate dielectrics in lowvoltage organic transistors. ${ }^{45}$ Importantly, both SAMs can be processed from solution or vapor phase at room temperature onto large-area substrates and the resulting monolayers are known to exhibit excellent thermal stability making them compatible with the device process flowchart employed in this work. ${ }^{49}$ Similar to $\mathrm{Ga}_{2} \mathrm{O}_{3}$, HMDS and PHDA exhibit large bandgaps and as such are expected to give rise to multiple-eV quantum wells when incorporated as the barrier systems in $\mathrm{ZnO}$ quantum well-based RTDs. Unlike epitaxiallygrown and solution-grown tunneling barriers, however, the combination of the well-defined chemical structure (molecular length) and self-assembling character (defined by the anchoring chemical group used) of the SAMs enables control of the barrier width down to atomic dimensions through simple chemistry and highly scalable wet-based deposition techniques. 


\section{WILEY-VCH}

Figure 6(b) displays a schematic representation of the SAM-based device structure developed, while Figure 6(c) shows the idealized energy band diagram of the device under equilibrium at zero bias. Representative sets of the I-V characteristics measured for two devices based on HMDS and PHDA barrier layers are shown in Figure 6(d) and Figure 6(e), respectively. Here, five sets of $\mathrm{I}-\mathrm{V}$ characteristics were recorded sequentially from each device at room temperature. Both types of hybrid RTDs show well-defined NDC regions with peak-to-valley ratios in the range 1-2. The peak current densities measured just before the NDC region was on the order of $10 \mathrm{~A} / \mathrm{cm}^{2}$ for both types of devices and were recorded at around $\sim 1.2 \mathrm{~V}$ for PHDA and at $\sim 1.8 \mathrm{~V}$ for HMDS-based RTDs. The latter value is significantly lower than current densities reported for traditional RTDs (typically in the range 0.5-10 kA/cm) based on AlGaAs/GaAs, InAs/AlSb, InGaAs/AlAs/InAs etc. ${ }^{25,27,50} \mathrm{We}$ attribute this to a number of factors that include, the significantly lower electron mobility of the polycrystalline $\mathrm{ZnO}$ [Fig. $\mathrm{S} 1(\mathrm{c})]$ and the presence of inactive device regions i.e. parts of the apparent device area that do not exhibit resonant tunneling conduction due to quantum well/barrier layer thickness variations.

The higher voltages with respect to those measured in the $\mathrm{Ga}_{2} \mathrm{O}_{3}$ based $\mathrm{ZnO}$ RTDs is in agreement with a deeper quantum well, ${ }^{42}$ resulting from the wide bandgap nature of these SAMs. Although the structural quality of our devices is not expected to be comparable in terms of uniformity to epitaxially grown RTDs, the use of conformal SAM barriers in combination with solution processed low-dimensional ZnO QWs appears to yield functional devices with operating characteristics closely resembling those of state-of-the-art GaAs/AlGaAs double-barrier RTDs. ${ }^{26}$ The improved electrical stability of the SAM-based (hybrid) $\mathrm{ZnO}$ RTDs with respect to $\mathrm{Ga}_{2} \mathrm{O}_{3}$ based devices is attributed to the conformal nature 


\section{WILEY-VCH}

of the SAM molecules. In the case of $\mathrm{Ga}_{2} \mathrm{O}_{3}$ barriers, certain intermixing between $\mathrm{ZnO}$ and $\mathrm{Ga}_{2} \mathrm{O}_{3}$ may take place leading to non-idealities in the barrier profile while the $\mathrm{Ga}_{2} \mathrm{O}_{3}$ barriers themselves are likely to exhibit structural roughness. On the contrary, SAM barriers are characterized by an extremely well-defined chemical structure and hence molecular length (i.e. barrier thickness) that is typically below $3 \mathrm{~nm}$ depending on the molecule used. Because of these unique characteristics, incorporation of organic SAMs as the tunneling barriers is expected to have a negligible impact on interfaces' roughness and stoichiometry.

Finally, we note that the qualitative difference in the form of the I-V curves measured from RTDs employing $\mathrm{Ga}_{2} \mathrm{O}_{3}$ barriers and those employing SAM barriers is a persistent characteristic of all devices measured in this study. We thus believe that the nature of the tunneling barriers is the primary reason for this observation and potentially a key to further developments. However, an in-depth study into the nature of this difference is beyond the scope of this work and will be the focus of future investigations.

\section{Conclusions}

We have presented strong evidence of the existence of quantized energy states in ultra-thin layers of $\mathrm{ZnO}$ processed from solution at $\leq 200^{\circ} \mathrm{C}$ in air. In agreement with experiments carried out using traditional quantum well systems (e.g. GaAs/AlGaAs ${ }^{8}$ ), we observe a widening of the optical band-gap of $\mathrm{ZnO}$ layers as the mean thickness is reduced, accompanied by a blue shift in the $\lambda_{\operatorname{MAx}}$ of the photoluminescence signal. By combining these solution-processed $\mathrm{ZnO}$ layers with $\mathrm{Ga}_{2} \mathrm{O}_{3}$ or organic self-assembled monolayers as tunneling barrier materials, two terminal devices with current-voltage characteristics resembling those of traditional double barrier resonant tunneling diodes were fabricated. The devices were 


\section{WILEY-VCH}

observed to exhibit reproducible negative differential conductivity at room temperature with peak-to-valley ratios in the range 2-7. The ability to grow from solution and at plasticcompatible temperatures $\left(\leq 200^{\circ} \mathrm{C}\right)$ complex heterostructures with nano-scale accuracy, and the demonstration of what appear to be functional resonant tunneling diodes with performance characteristics comparable to traditional GaAs/AlGaAs devices, ${ }^{26}$ creates new opportunities for both basic research and various technological applications.

\section{Experimental Section}

\section{Solution preparation and processing}

$\mathrm{ZnO}$ hydrate $\left(\mathrm{ZnO} \cdot \mathrm{xH}_{2} \mathrm{O}\right)$ was dissolved in ammonium hydroxide at a variety of concentrations. Gallium nitrate hydrate $\left(\mathrm{Ga}\left(\mathrm{NO}_{3}\right)_{3} \mathrm{xH}_{2} \mathrm{O}\right)$ was dissolved in deionized water at a concentration of $12 \mathrm{mg} / \mathrm{ml}$. Solutions were stirred overnight at room temperature before deposition. Solutions of phosphonohexadecanoic acid (PHDA) were prepared in IPA at a concentration of $50 \mathrm{mg} / 30 \mathrm{ml}$. Hexamethyldisilazane (HMDS) was purchased in solution and used as received. All materials are commercially available and were obtained from Sigma-Aldrich (UK).

\section{High-resolution transmission electron microscopy (HRTEM)}

A transmission electron microscope operating at an accelerating voltage of $300 \mathrm{kV}$ (Titan 80300 Super Twin, FEI Company) was used to acquire cross-section micrographs. Charged couple device (CCD) camera (Model: US4000, Gatan Inc.) was used to record HR-TEM images. Samples were prepared on a focused ion beam (FIB; Helios 400s, FEI) equipped with a nanomanipulator (Omniprobe, AutoProbe300) with lift-out method. Electron beam assisted 


\section{WILEY-VCH}

carbon and platinum deposition was performed on the sample surface to protect the thin film surface against the ion beam bombardment during ion beam milling. Ga ion beam $(30 \mathrm{kV}, 9$ $\mathrm{nA})$ was first used to cut the sample from the bulk $(30 \mathrm{kV}, 9 \mathrm{nA})$, after which it was attached to a $\mathrm{Cu}$ grid using a lift-out method. The sample was subsequently thinned down to ca. $50 \mathrm{~nm}$ thickness $(30 \mathrm{kV}, 93 \mathrm{pA})$ and cleaned $(2 \mathrm{kV}, 28 \mathrm{pA})$ to get rid of areas of the sample damaged during the thinning process.

\section{Grazing incident X-ray diffraction (GID)}

Grazing incident x-ray diffraction measurements were carried out on beamline G2 in Cornell High Energy Synchrotron Source (CHESS), Cornell University. The samples were aligned on a Kappa diffractometer with the X-ray energy of $13.65 \mathrm{keV}(\lambda=0.0908 \mathrm{~nm})$ through a Be single-crystal monochromator. The data was collected using a 640-element 1D diode-array detector, with a set of $0.1^{\circ}$ Soller slits mounted on the detector arm to provide an in-plane resolution of $0.16^{\circ}$. The grazing incident angle was fixed at $0.1^{\circ}$ in GID.

\section{Atomic force microscopy (AFM)}

Atomic force microscopy was carried out in tapping mode using an Agilent 5500 atomic force microscope in ambient atmosphere. The approximate resonance frequency of the cantilever was $250 \mathrm{kHz}$ and the force constant was approximately $60 \mathrm{Nm}^{-1}$.

\section{Optical transmittance measurements}

$\mathrm{ZnO}$ films were spin-cast onto quartz substrates from the solutions described above in air. The films were then annealed at $200{ }^{\circ} \mathrm{C}$ for 30 minutes in air. Measurements were carried out with 


\section{WILEY-VCH}

a Shimadzu UV-2600 ultraviolet-visible spectrophotometer. Transmittance and reflectance measurements were carried out for each sample. The transmittance corrected for reflectance was derived from the raw transmittance + raw reflectance.

\section{Photoluminescence measurements}

$\mathrm{ZnO}$ films were spin-cast onto polished silicon in air. The films were annealed at $200^{\circ} \mathrm{C}$ for 30 minutes in air. Low temperature photoluminescence (PL) spectra of the films were excited by a $35 \mathrm{~mW} \mathrm{He}-\mathrm{Cd}$ continuous wave laser emitting at $325 \mathrm{~nm}$. The measurements were performed at $22 \mathrm{~K}$ in high vacuum and the recorded spectra were resolved using a spectrometer with a UV grating of 600 grooves $/ \mathrm{mm}$ and a sensitive, calibrated liquid nitrogen cooled CCD camera.

\section{Resonant tunneling diodes fabrication}

All two-terminal resonant tunneling diode (RTD)-like structures were fabricated on glass substrates. In brief; $50 \mathrm{~nm}$-thick aluminum bottom electrodes were deposited via thermal evaporation under high vacuum through shadow masks at an average rate of $0.5 \AA^{-1}$. A bulk $\mathrm{ZnO}$ layer was deposited by 5 sequential spin-casting and thermal annealing steps. Each spincasting step was followed by an annealing step at $200{ }^{\circ} \mathrm{C}$ for 30 minutes in air. Next a barrier layer was deposited on top of the bottom bulk $\mathrm{ZnO}$ layer. Three different materials were employed as tunneling barriers in three different sets of devices. For $\mathrm{Ga}_{2} \mathrm{O}_{3}$-based devices, a single layer of $\mathrm{Ga}_{2} \mathrm{O}_{3}$ was spin-cast from gallium nitrate hydrate solution in air. The samples were then annealed at $200{ }^{\circ} \mathrm{C}$ for 30 minutes in air. For the second set of devices, HMDS was applied as a barrier layer. This was applied by placing the samples in vapor-phase HMDS at $80{ }^{\circ} \mathrm{C}$ for 30 minutes. Finally a monolayer of PHDA was applied to the third set of devices by 


\section{WILEY-VCH}

submerging the samples in PHDA solution for 90 minutes each. The PHDA-based samples were then thoroughly rinsed in IPA before being annealed in nitrogen at $90{ }^{\circ} \mathrm{C}$ for 30 minutes. A single ultra-thin layer of $\mathrm{ZnO}$ was then spin-cast onto the pre-deposited tunneling barrier layer acting as the quantum well. The layer thickness of the $\mathrm{ZnO}$ quantum well was estimated to be on the order of $\sim 2.4 \mathrm{~nm}$ and was controlled by tuning the concentration of the precursor solution and spinning speed during depositing. A second barrier layer of either $\mathrm{Ga}_{2} \mathrm{O}_{3}, \mathrm{HMDS}$ or PHDA was then applied on top of the thin $\mathrm{ZnO}$ layer in an identical manner as described above. A second bulk ZnO layer was deposited directly onto the second barrier layer via 5sequential spin-castings/thermal annealing steps, as described above for the bottom bulk $\mathrm{ZnO}$ layer. Finally, $50 \mathrm{~nm}$-thick aluminum top electrodes were deposited via thermal evaporation under high vacuum through shadow masks at an average rate of $0.5 \AA \mathrm{s}^{-1}$. Electrical characterization was carried out at room temperature under ambient pressure in nitrogen using an Agilent B2902A semiconductor parameter analyzer. The device areas of as-prepared devices were in the range $0.1-0.64 \mathrm{~mm}^{2}$.

\section{$X$-ray photoelectron spectroscopy and ultraviolet photoelectron spectroscopy}

Surface analysis studies were performed in an ultra-high vacuum system (UHV) which consists of a fast entry specimen assembly, a sample preparation and an analysis chamber equipped with a SPECS LHS-10 hemispherical electron analyzer. The base pressure in both chambers was $1 \times 10^{-9}$ mbar. Unmonochromatized AlKa line at $1486.6 \mathrm{eV}$ and an analyzer pass energy of $36 \mathrm{eV}$, giving a full width at half maximum (FWHM) of $0.9 \mathrm{eV}$ for the $\mathrm{Au} 4 \mathrm{f}_{7 / 2}$ peak, were used in all X-ray photoelectron spectroscopy (XPS) measurements. The analyzed area was an ellipse with dimensions $2.5 \times 4.5 \mathrm{~mm}^{2}$. The XPS core level spectra were analyzed using a fitting routine, which can decompose each spectrum into individual mixed Gaussian- 


\section{WILEY-VCH}

Lorentzian peaks after a Shirley background subtraction. The samples were measured as received. The main $\mathrm{C} 1 \mathrm{~s}$ peak at $284.8 \mathrm{eV}$ from superficial carbon was used in binding energy (BE) corrections for specimen charging. The ultraviolet photoelectron spectroscopy (UPS) spectra were obtained using HeI irradiation with $\mathrm{h} v=21.23 \mathrm{eV}$ produced by a UV source (model UVS 10/35). During UPS measurements the analyzer was working at the Constant Retarding Ratio $(\mathrm{CRR})$ mode, with $\mathrm{CRR}=10$. The work function $(\Phi)$ was determined from the UPS spectra by subtracting their width (i.e. the energy difference between the analyzer Fermi level and the high binding energy cut-off), from the HeI excitation energy. For these measurements a bias of $-12.29 \mathrm{~V}$ was applied to the sample in order to avoid interference of the spectrometer threshold in the UPS spectra.

\section{Supporting Information}

Supporting Information is available from the Wiley Online Library or from the author.

\section{Acknowledgements}

J.G.L. Y.H.L. J.S. and T.D.A. are grateful to Dutch Polymer Institute (DPI) S-PLORE grant no. 735 and European Research Council (ERC) AMPRO project no. 280221 for financial support. CHESS is supported by the NSF \& NIH/NIGMS via NSF award DMR-1332208.

Received: ((will be filled in by the editorial staff))

Revised: ((will be filled in by the editorial staff)) Published online: ((will be filled in by the editorial staff)) 


\section{WILEY-VCH}

\section{References}

[1] E. Fortunato, P. Barquinha, R. Martins, Adv Mater 2012, 24, 2945.

[2] S. R. Thomas, P. Pattanasattayavong, T. D. Anthopoulos, Chem Soc Rev 2013, 42, 6910.

[3] K. Ghaffarzadeh, R. Das, http:/www.idtechex.com/research/reports/metal-oxide-tftbackplanes-for-displays-2013-2018-analysis-trends-forecasts-000334.asp?viewopt=desc 2013.

[4] K. K. Banger, Y. Yamashita, K. Mori, R. L. Peterson, T. Leedham, J. Rickard, H. Sirringhaus, Nat Mater 2011, 10, 45.

[5] R. Theissmann, S. Bubel, M. Sanlialp, C. Busch, G. Schierning, R. Schmechel, Thin Solid Films 2011, 519, 5623.

[6] Y.-H. Lin, H. Faber, K. Zhao, Q. Wang, A. Amassian, M. McLachlan, T. D. Anthopoulos, Adv Mater 2013, 25, 4340.

[7] J. S. Lee, Y.-J. Kwack, W.-S. Choi, Journal of Korean Physical Society 2011, 59, 3055.

[8] R. Dingle, W. Wiegmann, C. H. Henry, Phys Rev Lett 1974, 33, 827.

[9] G. Adamopoulos, A. Bashir, W. P. Gillin, S. Georgakopoulos, M. Shkunov, M. A.

Baklar, N. Stingelin, D. D. C. Bradley, T. D. Anthopoulos, Adv Funct Mater 2011, 21, 525.

[10] A. I. M. Rae, Quantum mechanics, Taylor \& Francis Group, 2008.

[11] Y. H. Lin, H. Faber, E. Stratakis, L. Sygellou, E. Kymakis, N. A. Hastas, L. R., K. Zhao, A. Amassian, N. D. Treat, M. McLachlan, T. D. Anthopoulos, In Press. 2014.

[12] C. Weisbuch, B. Vinter, Quantum Semiconductor Structures: Fundamentals and Applications, Academic Press, 1991.

[13] J. R. Hook, H. E. Hall, Solid State Physics, Wiley, 1991.

[14] B. Enright, D. Fitzmaurice, The Journal of Physical Chemistry 1996, 100, 1027. 


\section{WILEY-VCH}

[15] J. Tauc, R. Grigorovici, A. Vancu, physica status solidi (b) 1966, 15, 627.

[16] J. Tauc, Materials Research Bulletin 1968, 3, 37.

[17] Y. Xu, M. A. A. Schoonen, American Mineralogist 2000, 85, 543.

[18] W. S. Baer, Physical Review 1967, 154, 785.

[19] F. Torricelli, J. R. Meijboom, E. Smits, A. K. Tripathi, M. Ferroni, S. Federici, G. H.

Gelinck, L. Colalongo, Z. M. Kovacs-Vajna, D. de Leeuw, E. Cantatore, Electron Devices, IEEE Transactions on 2011, 58, 2610.

[20] K. Abe, K. Nomura, T. Kamiya, H. Hosono, Phys Rev B 2012, 86, 081202.

[21] S. T. Tan, B. J. Chen, X. W. Sun, W. J. Fan, H. S. Kwok, X. H. Zhang, S. J. Chua, J Appl Phys 2005, 98.

[22] A. A. Mosquera, D. Horwat, A. Rashkovskiy, A. Kovalev, P. Miska, D. Wainstein, J. M. Albella, J. L. Endrino, Sci. Rep. 2013, 3.

[23] C. Dang, J. Lee, C. Breen, J. S. Steckel, S. Coe-Sullivan, A. Nurmikko, Nat Nano $2012,7,335$.

[24] L. E. Brus, The Journal of Chemical Physics 1984, 80, 4403.

[25] L. L. Chang, L. Esaki, R. Tsu, Appl Phys Lett 1974, 24, 593.

[26] T. C. L. G. Sollner, W. D. Goodhue, P. E. Tannenwald, C. D. Parker, D. D. Peck, Appl Phys Lett 1983, 43, 588.

[27] E. Ozbay, D. M. Bloom, D. H. Chow, J. N. Schulman, Electron Device Letters, IEEE 1993, 14, 400 .

[28] K. Ismail, B. S. Meyerson, P. J. Wang, Appl Phys Lett 1991, 59, 973.

[29] H. Mizuta, T. Tanoue, The Physics and Applications of Resonant Tunnelling Diodes, Cambridge University Press, 2006. 


\section{WILEY-VCH}

[30] L. Britnell, R. V. Gorbachev, A. K. Geim, L. A. Ponomarenko, A. Mishchenko, M. T.

Greenaway, T. M. Fromhold, K. S. Novoselov, L. Eaves, Nat Commun 2013, 4, 1794.

[31] S. Krishnamoorthy, A. A. Iliadis, A. Inumpudi, S. Choopun, R. D. Vispute, T.

Venkatesan, Solid-State Electronics 2002, 46, 1633.

[32] J. Chen, M. A. Reed, A. M. Rawlett, J. M. Tour, Science 1999, 286, 1550.

[33] J. L. Huber, J. Chen, J. A. McCormack, C. W. Zhou, M. A. Reed, Electron Devices, IEEE Transactions on 1997, 44, 2149.

[34] J. Faist, F. Capasso, D. L. Sivco, C. Sirtori, A. L. Hutchinson, A. Y. Cho, Science 1994, 264, 553.

[35] B. S. Williams, Nat Photon 2007, 1, 517.

[36] L. D. Hicks, M. S. Dresselhaus, Phys Rev B 1993, 47, 12727.

[37] S. R. Thomas, G. Adamopoulos, Y.-H. Lin, H. Faber, L. Sygellou, E. Stratakis, N. Pliatsikas, P. A. Patsalas, T. D. Anthopoulos, Appl Phys Lett 2014, 105.

[38] A. Janotti, C. G. Van de Walle, Phys Rev B 2007, 76, 165202.

[39] A. Dal Corso, M. Posternak, R. Resta, A. Baldereschi, Phys Rev B 1994, 50, 10715.

[40] Y. Li, S. Long, Q. Liu, H. Lü, S. Liu, M. Liu, Chin. Sci. Bull. 2011, 56, 3072.

[41] S. Nigo, M. Kubota, Y. Harada, T. Hirayama, S. Kato, H. Kitazawa, G. Kido, J Appl Phys 2012, 112.

[42] R. Tsu, L. Esaki, Appl Phys Lett 1973, 22, 562.

[43] M. L. Hair, W. Hertl, The Journal of Physical Chemistry 1971, 75, 2181.

[44] R. Anwander, I. Nagl, M. Widenmeyer, G. Engelhardt, O. Groeger, C. Palm, T. Röser, The Journal of Physical Chemistry B 2000, 104, 3532.

[45] J. M. Ball, P. H. Wobkenberg, F. Colleaux, M. Heeney, J. E. Anthony, I. McCulloch, D. D. C. Bradley, T. D. Anthopoulos, Appl Phys Lett 2009, 95. 


\section{WILEY-VCH}

[46] M. Novak, T. Schmaltz, H. Faber, M. Halik, Appl Phys Lett 2011, 98.

[47] L.-L. Chua, J. Zaumseil, J.-F. Chang, E. C. W. Ou, P. K. H. Ho, H. Sirringhaus, R. H. Friend, Nature 2005, 434, 194.

[48] S. C. Lim, S. H. Kim, J. H. Lee, M. K. Kim, D. J. Kim, T. Zyung, Synthetic Met 2005, $148,75$.

[49] A. Bashir, P. H. Wöbkenberg, J. Smith, J. M. Ball, G. Adamopoulos, D. D. C. Bradley, T. D. Anthopoulos, Adv Mater 2009, 21, 2226.

[50] C. I. Huang, M. J. Paulus, C. A. Bozada, S. C. Dudley, K. R. Evans, C. E. Stutz, R. L. Jones, M. E. Cheney, Appl Phys Lett 1987, 51, 121. 


\section{WILEY-VCH}
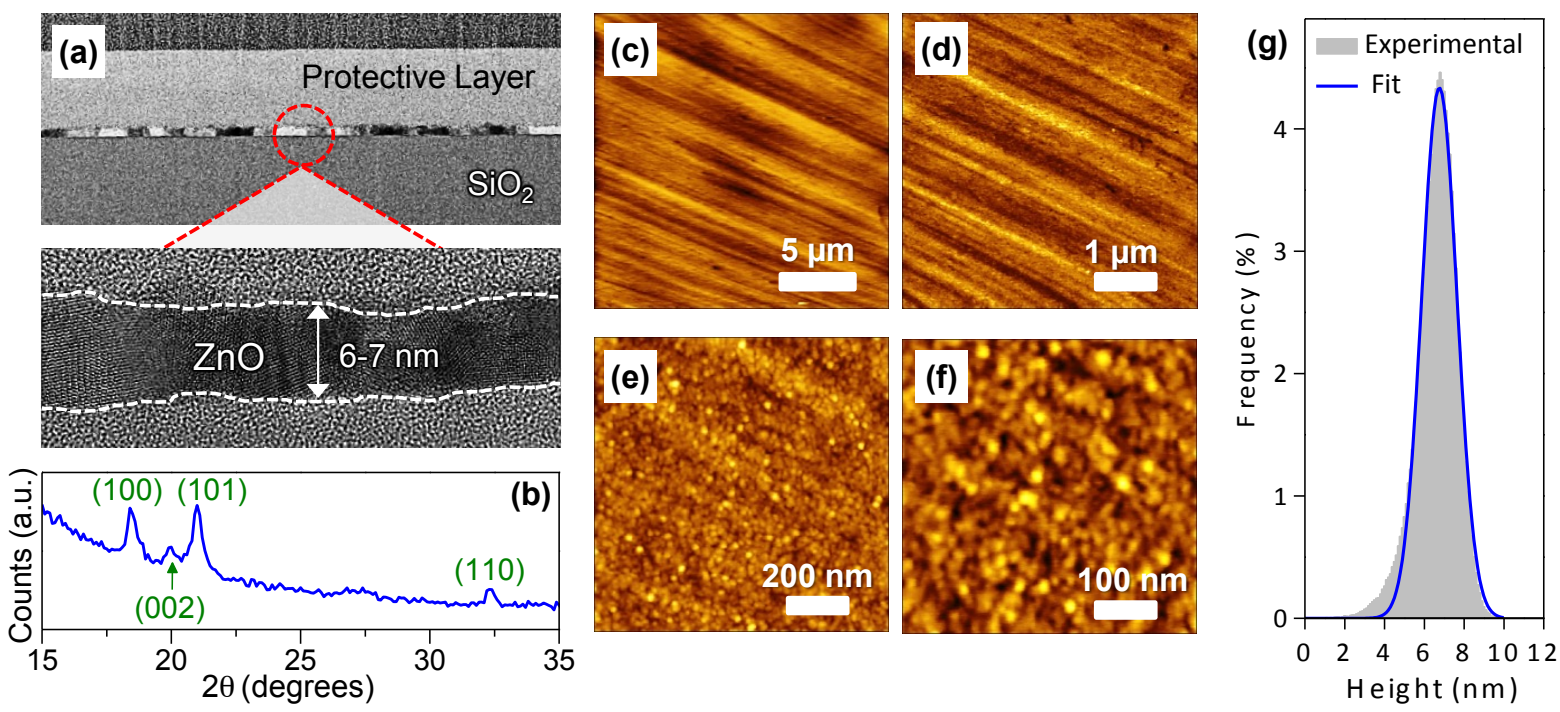

Figure 1. (a) High-resolution transmission electron microscope (HRTEM) cross-sectional images (medium and high magnification) of a $\mathrm{ZnO}$ layer spin-coated on $\mathrm{SiO}_{2}$. (b) Grazing incident X-ray diffraction (GID) spectra of similar $\mathrm{ZnO}$ film on $\mathrm{SiO}_{2}$ substrate. (c)-(f) Tapping-mode atomic force microscopy (AFM) images of thin $\mathrm{ZnO}$ film deposited onto quartz substrate. (g) Surface height distribution extracted from the AFM image in (e). The solid line is a Gaussian distribution fitted to the experimental distribution, with a mean of 6.8 $\mathrm{nm}$ and a standard deviation of $0.9 \mathrm{~nm}$. 


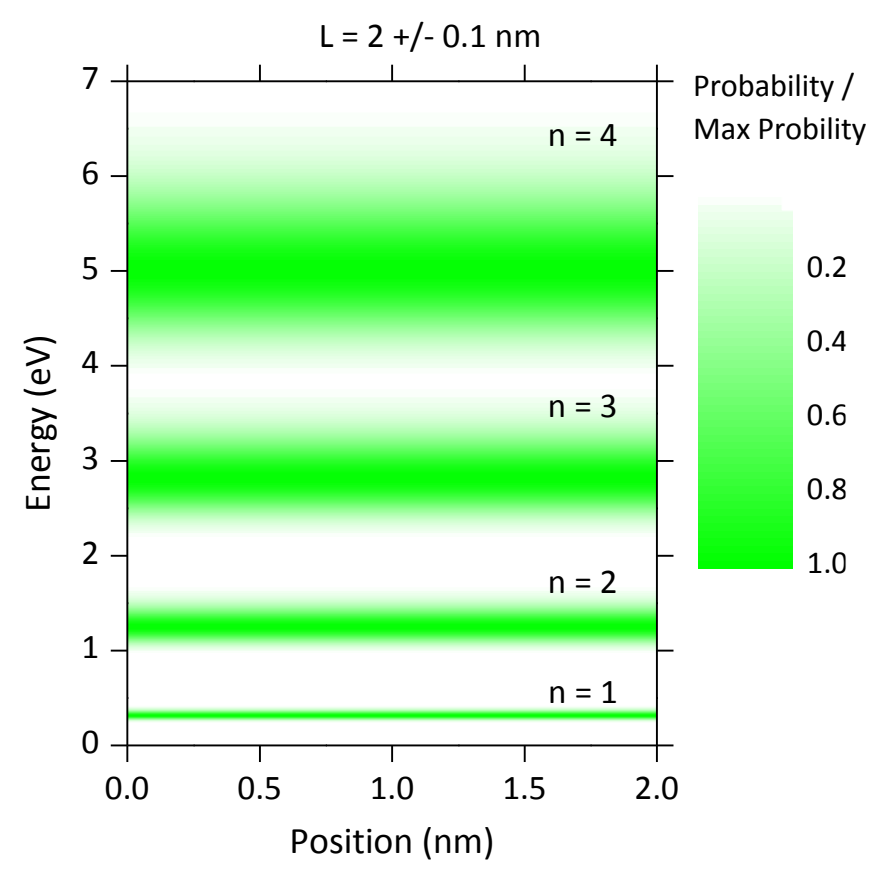

Figure 2. Probability distribution of lowest 4 energy states of infinite quantum well with a mean thickness of $2 \mathrm{~nm}$, a thickness standard deviation of $0.1 \mathrm{~nm}$ and an effective electron mass of $0.29 m_{e}$, evaluated using Eq. 2. Green represents the most likely energy for a given sub-band state and white represents an energy level for that state with a probability of zero. 
(a)

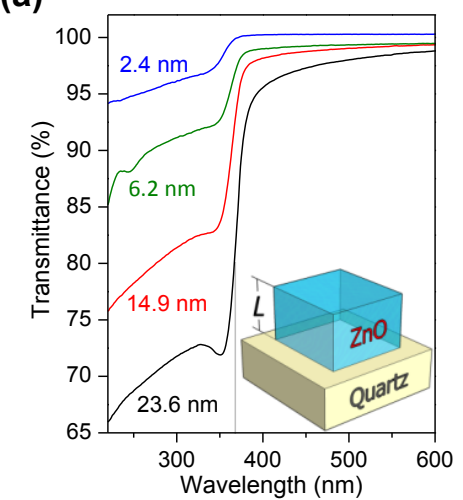

(c)

(b)
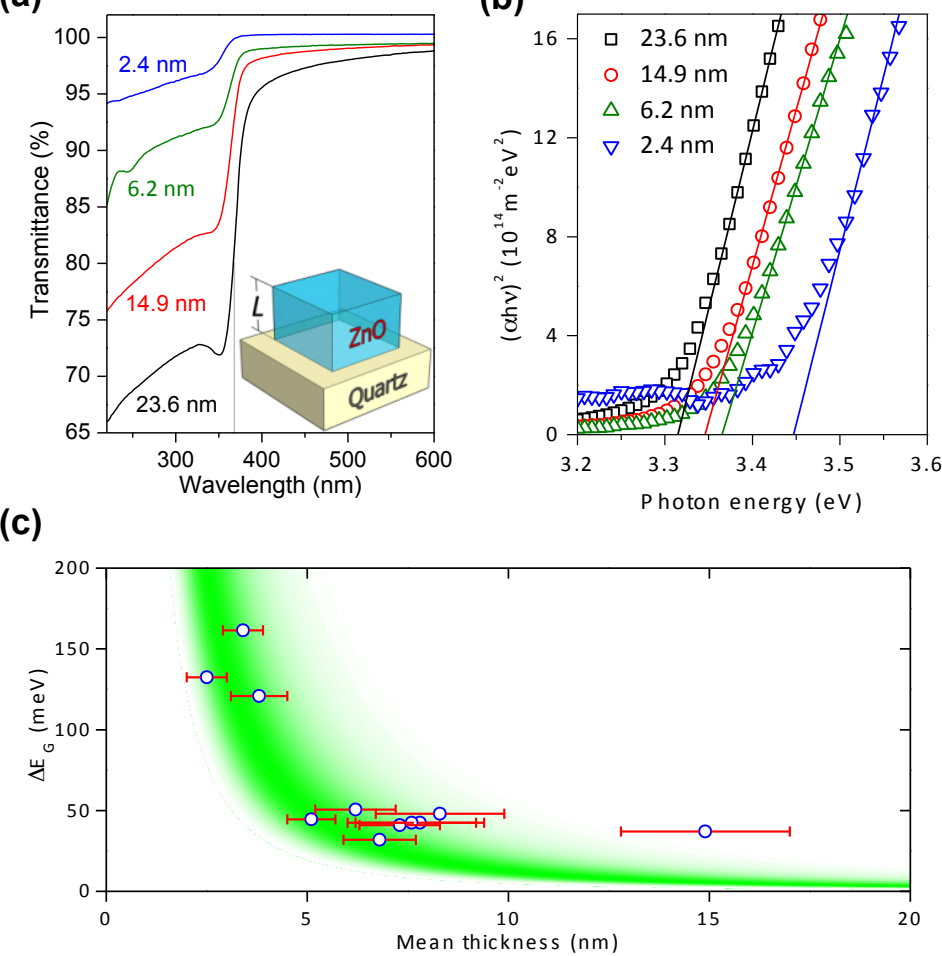

(d)

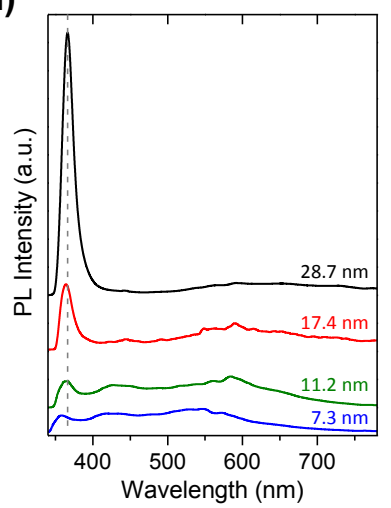

(e)

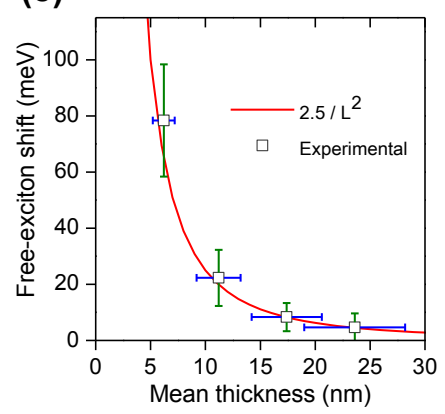

Figure 3. (a) Optical transmittance spectra of $4 \mathrm{ZnO}$ films of various thickness $(L)$ spin-cast on quartz, corrected for reflection. (b) Tauc plots of the $4 \mathrm{ZnO}$ films from (a). (c) Change in optical band gap approximated by Tauc analysis (see Supporting Information Section S2) against mean film thickness for $10 \mathrm{ZnO}$ films with respect to that of bulk $\mathrm{ZnO}$ (here approximated as a film $23.6 \mathrm{~nm}$ thick). The error bars represent the standard deviation in the film thickness. The green band illustrates the calculated change in band gap for an infinite quantum well with a standard deviation / mean ratio of 0.2. Green represents the energy with highest probability for a given mean well thickness and white represents an energy with probability zero. (d) Photoluminescence (PL) spectra of 4 thin $\mathrm{ZnO}$ films of various thickness spin-cast onto polished silicon, measured at $22 \mathrm{~K}$. (e) Shift in exciton energy with respect to bulk $\mathrm{ZnO}$ (here approximated as a film $28.7 \mathrm{~nm}$ thick) of 4 thin $\mathrm{ZnO}$ films deposited onto polished silicon, as a function of mean film thickness. Error bars on the $x$-axis represent the standard deviation in the film thickness. The solid line is a plot of $A / L^{2}$ fitted to the experimental data with a prefactor of $A=2.5 \mathrm{meVnm}^{2}$. 
(a)

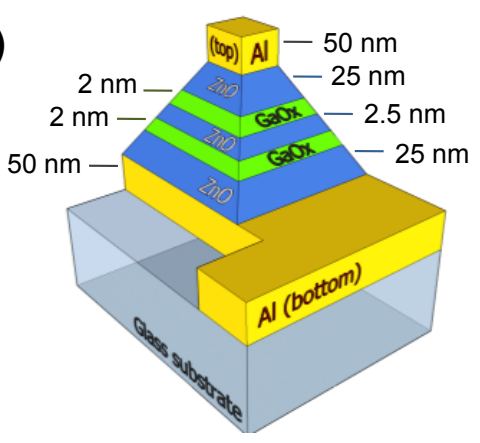

(b)

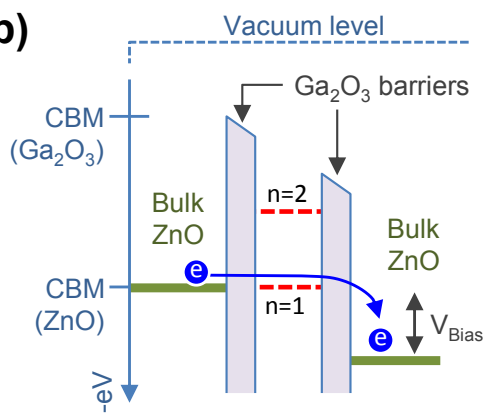

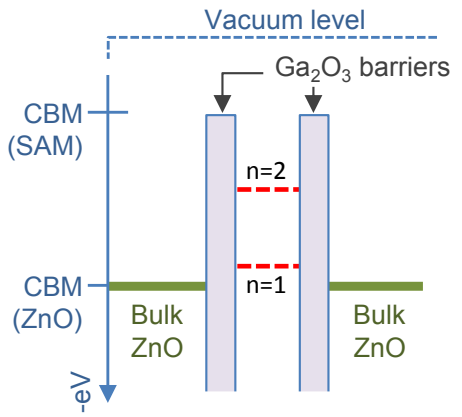

(c)

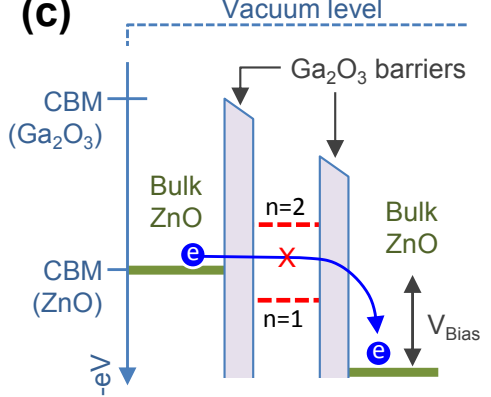

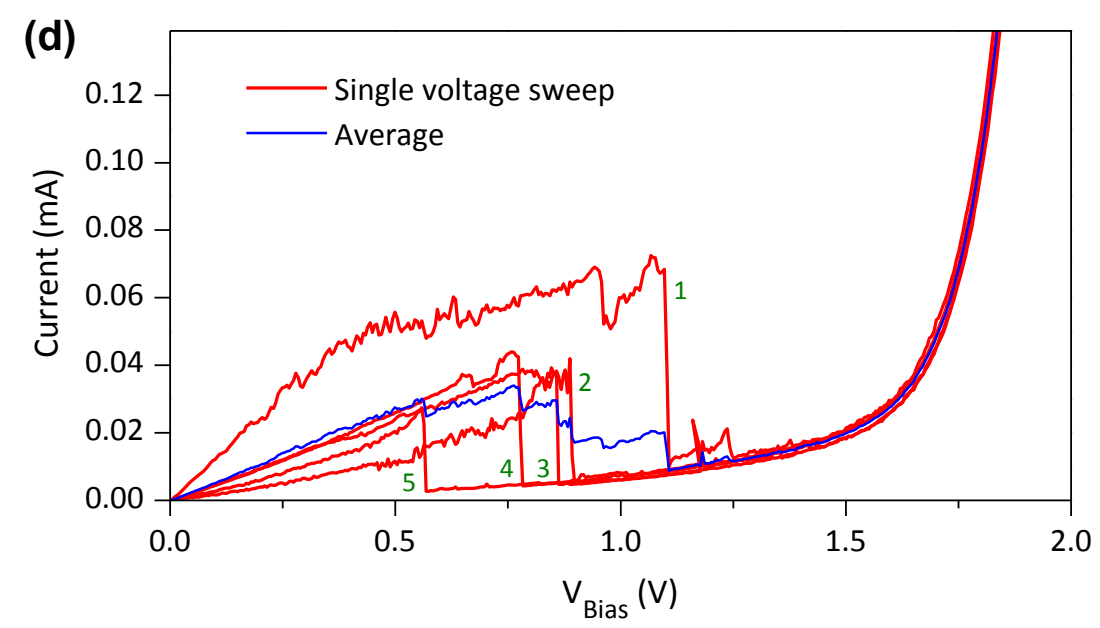

Figure 4. (a) Schematic representation of the solution-processed $\mathrm{ZnO}$ resonant tunnelingdiode (RTD) structure developed and the idealized energy band diagram under zero bias. The RTD consists of a central quantum well, formed of an ultra-thin $(\sim 2-3 \mathrm{~nm}) \mathrm{ZnO}$ layer confined between two ultra-thin $(\sim 2 \mathrm{~nm}) \mathrm{Ga}_{2} \mathrm{O}_{3}$ barrier layers. The quantum well structure is enclosed between two bulk ( $\sim 25 \mathrm{~nm}$-thick) $\mathrm{ZnO}$ layers on either side, which are in directcontact with aluminum electrodes (labeled Al). (b) and (c) Idealized representation of conduction band of solution-processed resonant tunneling-diode under two biasing conditions. The labels $n=1$ and $n=2$ represent the discreet energy levels of the central $\mathrm{ZnO}$ 


\section{WILEY-VCH}

semiconductor. The labels on the $y$-axis " $\mathrm{CBM}\left(\mathrm{Ga}_{2} \mathrm{O}_{3}\right)$ " and "CBM $(\mathrm{ZnO})$ " represent the energies of the conduction band minima of the $\mathrm{Ga}_{2} \mathrm{O}_{3}$ barrier layers and $\mathrm{ZnO}$ bulk layers respectively. (d) Current-voltage characteristics of a representative RTD, measured sequentially 5 times where curve 1 represents the first voltage sweep and curve 5 the last). The blue line is the average of the 5 measured curves (red). The device areas were $0.64 \mathrm{~mm}^{2}$ in each case and all measurements were carried out at room temperature under ambient pressure in nitrogen atmosphere. 
(a)
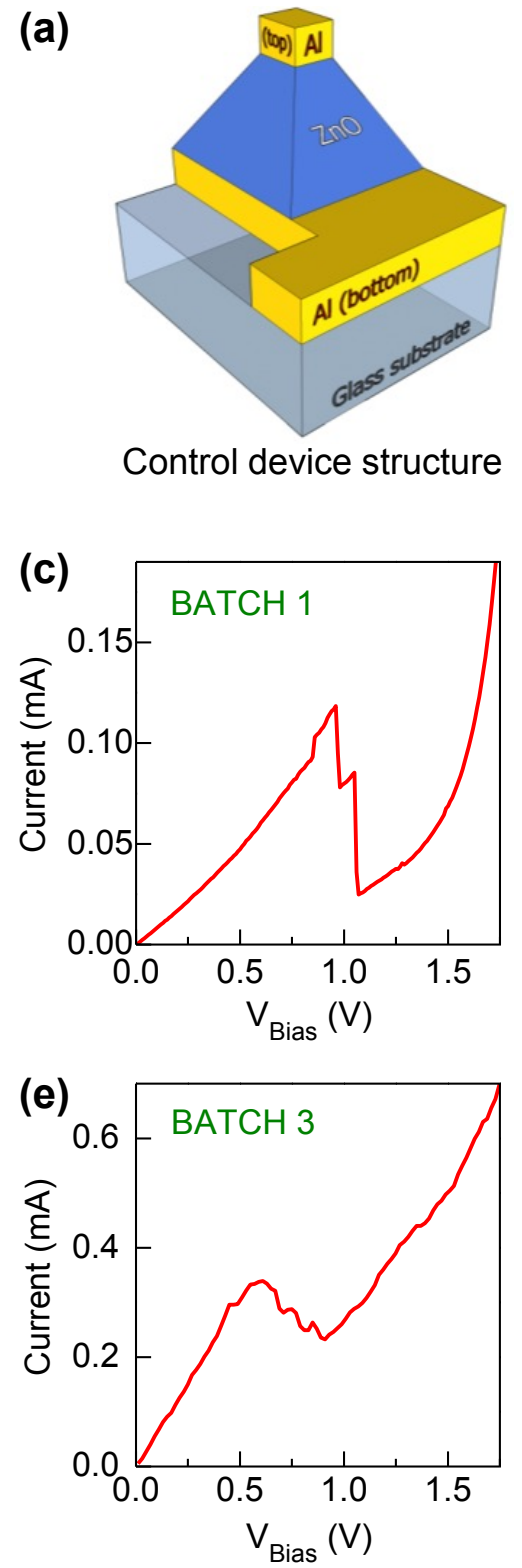
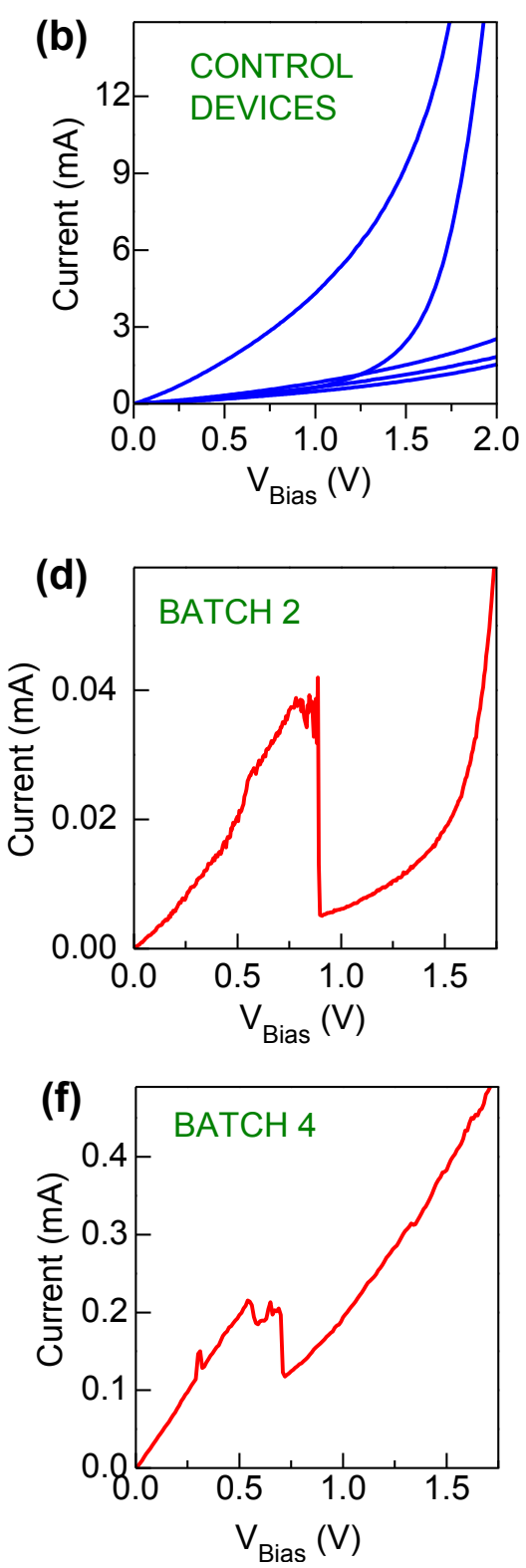

Figure 5. (a) Schematic representation of the control device structure developed. (b) Representative current-voltage characteristics of 5 example control devices, fabricated in parallel to the RTDs measured in Figure 4, but with the two $\mathrm{Ga}_{2} \mathrm{O}_{3}$ deposition steps omitted. The $\mathrm{ZnO}$ layer thickness used in all control devices was approximatley $50 \mathrm{~nm}$. (c)-(f) Representative current-voltage characteristics measured for 4 different $\mathrm{Ga}_{2} \mathrm{O}_{3}$-based resonanttunneling diodes prepared in four different batches on different dates. All RTDs consist of a central quantum well formed of an ultra-thin $(\sim 2-3 \mathrm{~nm}) \mathrm{ZnO}$ layer sandwiched between two ultra-thin $(\sim 2 \mathrm{~nm}) \mathrm{Ga}_{2} \mathrm{O}_{3}$ barrier layers. Device areas were $0.64 \mathrm{~mm}^{2}$ in each case and all measurements were carried out under ambient pressure in nitrogen. 
(a)

$$
\begin{gathered}
\mathrm{Si}^{-\mathrm{N}} \mathrm{Si}^{-} \\
\text {HMDS }
\end{gathered}
$$

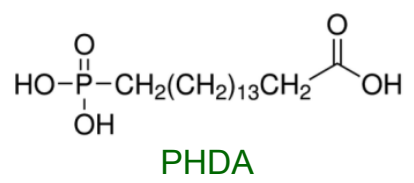

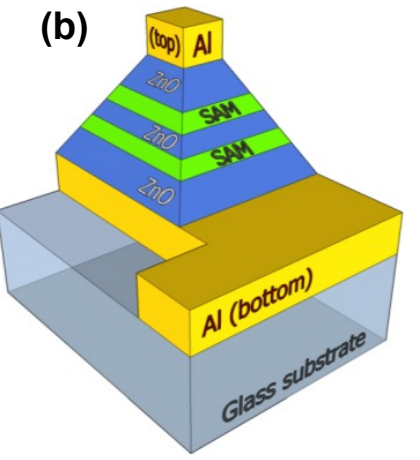
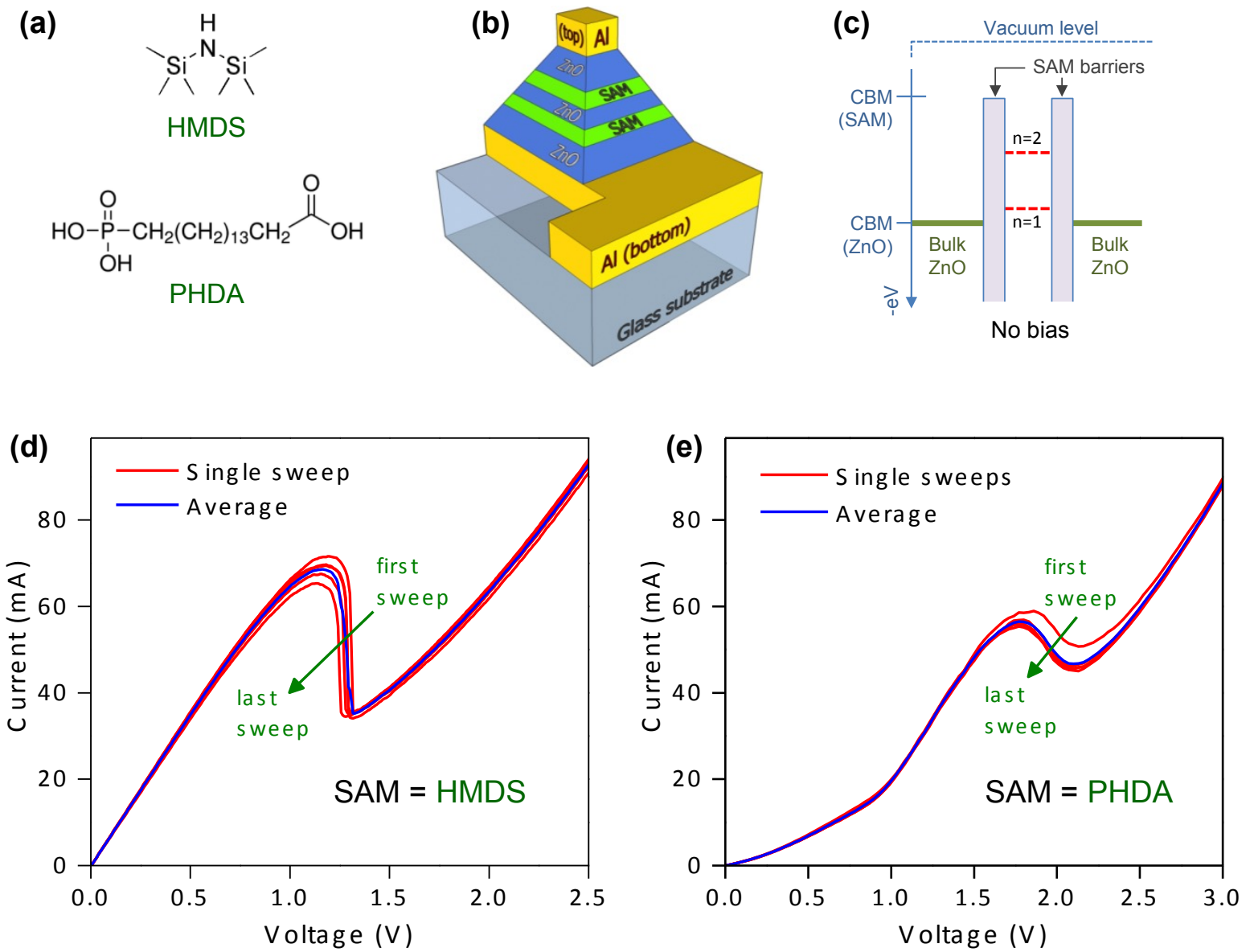

Figure 6. (a) Molecular structures of the organic barrier molecules hexamethyldisilazane (HMDS) and phosphonohexadecanoic acid (PHDA). (b) Schematic representation of solutionprocessed $\mathrm{ZnO}$ resonant tunneling-diode (RTD) structure employing self-assembled monolayer (SAM) barriers. The structure is identical to that of Figure 4(a) but with the $\mathrm{Ga}_{2} \mathrm{O}_{3}$ barriers being replaced by the SAM layers. (c) Idealized representation of conduction band of a SAM-based RTD without any bias. The labels on the $y$-axis "CBM (SAM)" and "CBM $(\mathrm{ZnO})$ " represent the energies of the conduction band minima of the SAM barrier monolayers and $\mathrm{ZnO}$ bulk layers respectively. Representative current-voltage characteristics measured for RTDs based on the barrier layers of (d) HMDS and (e) PHDA, measured sequentially 5 times. The blue line is the average of the five I-V characteristics (red curves). Each RTD consists of a 2-3 nm-thick $\mathrm{ZnO}$ quantum well confined between the two SAM barriers. The device areas were $0.64 \mathrm{~mm}^{2}$ in each case and all measurements were carried out at room temperature under ambient pressure in nitrogen atmosphere. 


\section{WILEY-VCH}

\section{The Table of Contents Entry}

The concept of quantized energy states in ultra-thin solution-processed zinc oxide $(\mathrm{ZnO})$ layers is explored. As-deposited layers are found to exhibit a characteristic widening of the energy bandgap with reducing thickness in accordance with theoretical predictions. When the $\mathrm{ZnO}$ layers are used as quantum-wells in carefully engineered two-terminal electronic devices, negative differential conductance is observed.

Keyword: Solution-Processed Semiconductors, Semiconducting Oxides, Energy Quantization, Resonant Tunneling Diodes, Zinc Oxide

John G. Labram*, Yen-Hung Lin, Kui Zhao, Ruipeng Li, Stuart R. Thomas, James Semple, Maria Androulidaki, Lamprini Sygellou, Martyn McLachlan, Emmanuel Stratakis, Aram Amassian, Thomas D. Anthopoulos*

Signatures of Quantized Energy States in Solution-Processed Ultra-Thin Layers of Metal Oxide Semiconductors and Their Devices

\section{ToC Figure}

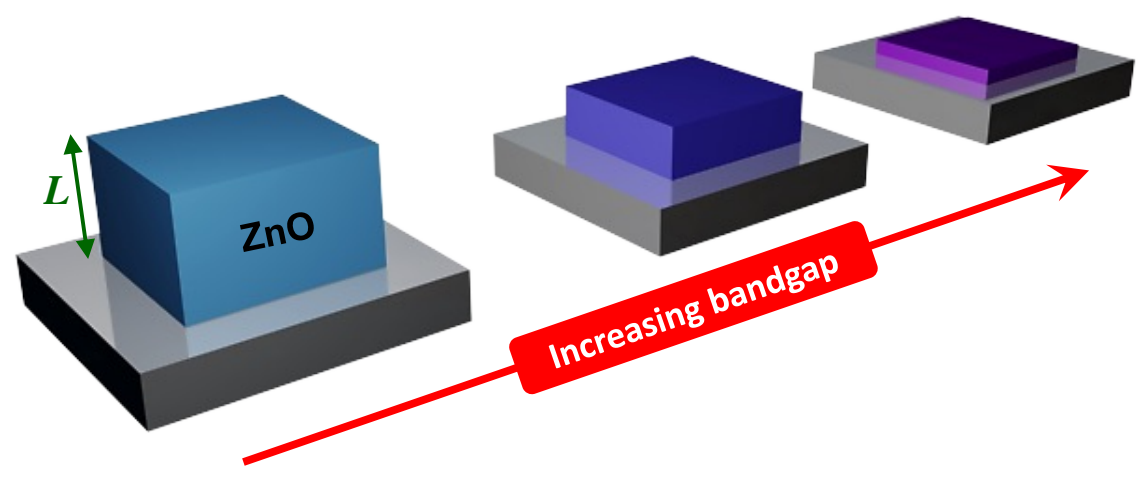

Copyright WILEY-VCH Verlag GmbH \& Co. KGaA, 69469 Weinheim, Germany, 2013. 


\section{WILEY-VCH}

\section{Supporting Information}

\section{Signatures of Quantized Energy States in Solution-Processed Ultra-Thin Layers of Metal Oxide Semiconductors and Their Devices}

John G. Labram*, Yen-Hung Lin, Kui Zhao, Ruipeng Li, Stuart R. Thomas, James Semple, Maria Androulidaki, Lamprini Sygellou, Emmanuel Stratakis, Aram Amassian, Martyn McLachlan, Thomas D. Anthopoulos*

*Correspondence to: E-mail: j.labram@imperial.ac.uk; t.anthopoulos@imperial.ac.uk

\section{S1. ZnO thin-film transistor measurements}

To demonstrate the continuousness of the as-grown $\mathrm{ZnO}$ films, bottom-gate, top-contact (BGTC) field-effect transistors were fabricated from $\mathrm{ZnO}$ deposited via identical processing methods as those deposited onto quartz (see main text). Figure S1(a) shows the transfer characteristics of a device with an active layer thickness of $3.8 \pm 0.7 \mathrm{~nm}$, and channel length and width of $200 \mu \mathrm{m}$ and $1.5 \mathrm{~mm}$ respectively. Figure S1(b) shows the output characteristics of the same device. The extracted field-effect electron mobility for this device is $0.2 \mathrm{~cm}^{2} \mathrm{~V}^{-1} \mathrm{~s}^{-1}$. Thicker films were observed to have slightly higher mobilities [Figure S1(c)], which is attributed to improved percolation of charges in the film.
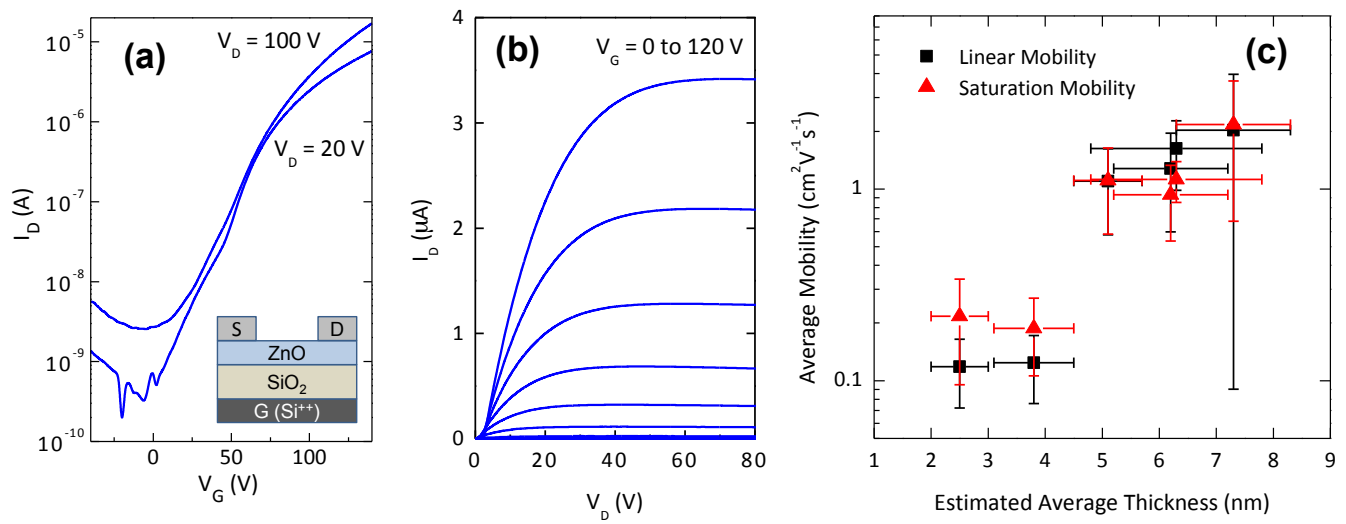


\section{WILEY-VCH}

Figure S1. (a) Transfer and (b) output characteristics of bottom-gate top-contact (BG-TC) $\mathrm{ZnO}$ transistor measured at room temperature in nitrogen at atmospheric pressure The mean film thickness of the $\mathrm{ZnO}$ layers used in this device was $\sim 3.8 \mathrm{~nm}$. The transistor had a channel length and width of $200 \mu \mathrm{m}$ and $1.5 \mathrm{~mm}$, respectively, and the geometrical capacitance of the channel was $8.6 \mathrm{nFcm}^{-2}$. Inset: Schematic diagram of field-effect transistor. The $\mathrm{S}$ and $\mathrm{D}$ indicate the aluminum source and drain electrodes and $\mathrm{G}$ the doped $\mathrm{Si}^{++}$gate electrode. (c) Average field-effect mobility of $5 \mathrm{BG}-\mathrm{TC} \mathrm{ZnO}$ transistors as a function of $\mathrm{ZnO}$ layer thickness.

\section{S2. Tauc analysis of solution-processed $\mathrm{ZnO}$ layers}

Figure S2 shows the Tauc plots ${ }^{[1]}$ for 11 thin $\mathrm{ZnO}$ films deposited on quartz substrates using the same processing conditions described in the Experimental Section of the main text.
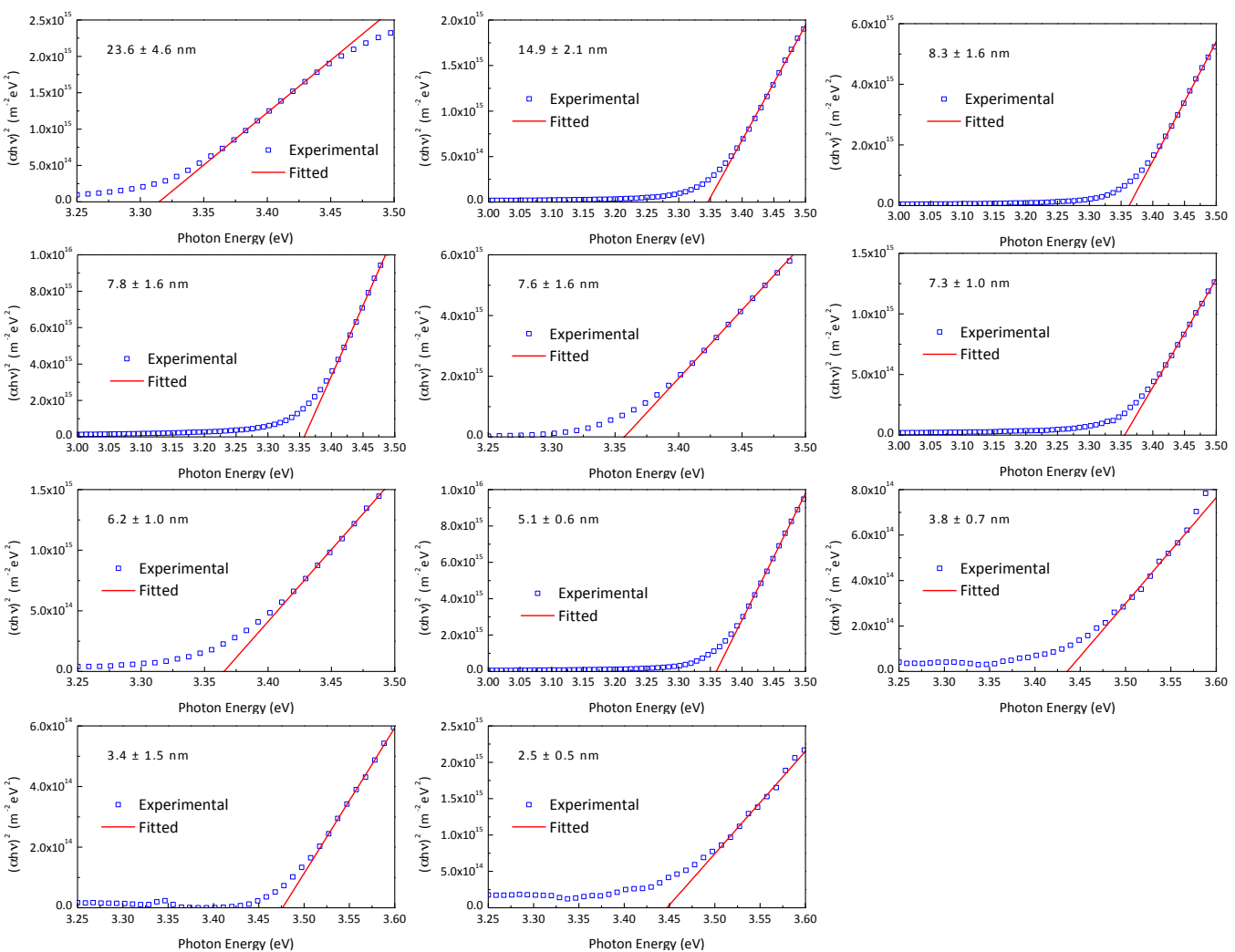

Photon Energy (eV) 


\section{WILEY-VCH}

Figure S2. Tauc plots for $11 \mathrm{ZnO}$ layers spin-cast on quartz with various film thicknesses. The mean film thickness with the standard deviation of each $\mathrm{ZnO}$ layer studied is shown in the top left corner of each plot.

\section{S3. Calculated electron transmission of resonant tunneling diodes}

The double-barrier resonant tunneling diode (RTD) structure can be considered to be an electron filter that permits the transmission of electrons at certain energies, whilst strongly attenuating their wavefunction amplitude at all other energies. By treating the system as an electron reflection and transmission problem, the transmission spectra of RTDs can be calculated. ${ }^{[2,3]}$ The RTD can be split into 5 regions (labelled $i$ ), separated by 4 interfaces labelled $z_{i}$, as illustrated in Figure S3(a). If the potential in region $i$ is $V_{i}$ the electron wavefunction in is this region can be described by Equations S1:

$\psi_{i}=A_{i} \exp \left(i k_{i} z\right)+B_{i} \exp \left(i k_{i} z\right)$

$k_{i}=\frac{\sqrt{\left(E-V_{i}\right) 2 m_{i}^{*}}}{\hbar}$

Here, $A_{i}$ and $B_{i}$ give the amplitude of the transmitted and reflected waves in each region respectively and $\hbar$ is the reduced Planck constant. In the Tsu and Esaki model the effective mass in each region is approximated to be constant at $m^{*}{ }^{[3]}$ 


\section{WILEY-VCH}

To conserve current at each interface we require that the wavefunctions and their first derivatives be equal. These boundary conditions lead to Eq. S2:

$$
\left(\begin{array}{l}
A_{i} \\
B_{i}
\end{array}\right)=\underline{\underline{R}}^{(i)}\left(\begin{array}{l}
A_{i+1} \\
B_{i+1}
\end{array}\right)=\left(\begin{array}{ll}
R_{11}{ }^{(i)} & R_{12}{ }^{(i)} \\
R_{21}{ }^{(i)} & R_{22}{ }^{(i)}
\end{array}\right)\left(\begin{array}{c}
A_{i+1} \\
B_{i+1}
\end{array}\right)
$$

Where the matrix elements of $\underline{\underline{R}}^{(i)}$ are given by Eq. S3:

$$
\begin{aligned}
& R_{11}{ }^{(i)}=\left(\frac{1}{2}+\frac{k_{i+1}}{k_{i}}\right) \exp \left[i\left(k_{i+1}-k_{i}\right) z_{i}\right] \\
& R_{12}^{(i)}=\left(\frac{1}{2}-\frac{k_{i+1}}{k_{i}}\right) \exp \left[-i\left(k_{i+1}+k_{i}\right) z_{i}\right] \\
& R_{21}{ }^{(i)}=\left(\frac{1}{2}-\frac{k_{i+1}}{k_{i}}\right) \exp \left[i\left(k_{i+1}+k_{i}\right) z_{i}\right] \\
& R_{22}^{(i)}=\left(\frac{1}{2}+\frac{k_{i+1}}{k_{i}}\right) \exp \left[-i\left(k_{i+1}-k_{i}\right) z_{i}\right]
\end{aligned}
$$

In the 5-region device in Figure S3(a), the relationship between the wavefunction amplitude in region 1 and region 5 can be described by Eq. S4:

$$
\left(\begin{array}{l}
A_{1} \\
B_{1}
\end{array}\right)=\underline{\underline{R}}^{(1)} \underline{\underline{R}}^{(2)} \underline{\underline{R}}^{(3)} \underline{\underline{R}}^{(4)}\left(\begin{array}{l}
A_{5} \\
B_{5}
\end{array}\right)=\underline{\underline{M}}\left(\begin{array}{l}
A_{5} \\
B_{5}
\end{array}\right)
$$




\section{WILEY-VCH}

It is clear from Figure S3(a) that for an electron incident in region 1 there cannot be any reflection in region 5 , hence $B_{5}=0$. The transmission probability is defined as the squared ratio of the transmitted electron flux to the incident flux, as given in Eq. S5:

$$
T=\frac{k_{1}}{k_{5}} \frac{\left|A_{5}\right|^{2}}{\left|A_{1}\right|^{2}}=\frac{k_{1}}{k_{5}} \frac{1}{\left|M_{11}\right|^{2}}
$$

This function has been calculated in Figure S3(b) for the ZnO RTD depicted in Figure 4(a) of the main text with $\mathrm{Ga}_{2} \mathrm{O}_{3}$ barrier layers. The effective mass of electrons in crystalline $\mathrm{ZnO}$

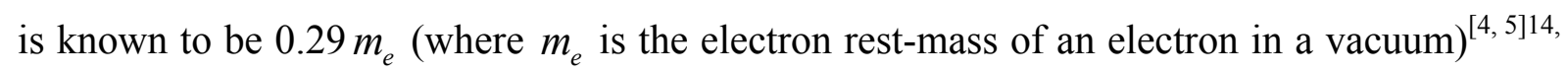
${ }^{18}$ and we have approximated the effective mass of electrons to be this value throughout the structure. The dimensions of the constituent layers in the structure were approximated from AFM measurements carried out on equivalent single films deposited onto quartz under identical processing conditions of those in the RTD. The thicknesses of regions 1, 2, 3, 4 and 5 were approximated to be $25 \mathrm{~nm}, 2 \mathrm{~nm}, 2.5 \mathrm{~nm}, 2 \mathrm{~nm}$ and $25 \mathrm{~nm}$, respectively. 
(a)
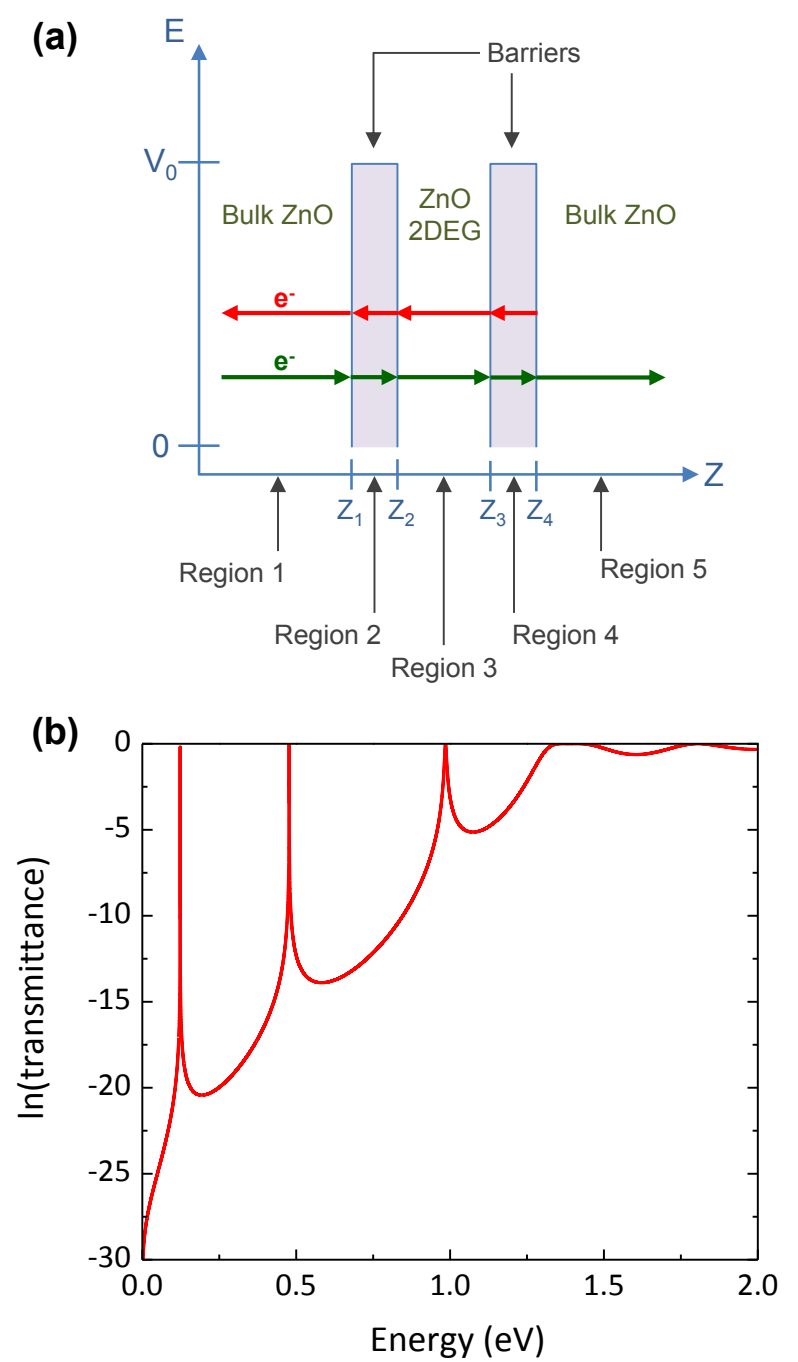

Figure S3. (a) Schematic representation of transmission and reflection problem in an idealized resonant tunneling diode (RTD). The energy of the system is set to $E=0$ at the conduction band minimum $(\mathrm{CBM})$ of bulk $\mathrm{ZnO} . V_{0}$ is the energy of the $\mathrm{CBM}$ of the barrier layers minus that of the $\mathrm{ZnO}$. The lower (green) arrows represent transmitted electrons and the upper (red) arrows represent reflected electrons. (b) Natural log of transmittance of idealized RTD calculated using Equation S5. An effective mass of $m^{*}=0.29 m_{e}$ is used as the effective mass of electrons throughout the system (where $m_{e}$ is the rest-mass of an electron in a vacuum), $V_{0}$ is set to $1.0 \mathrm{eV}$, and the length of regions $1-5$ are; $25 \mathrm{~nm}, 2 \mathrm{~nm}, 2.5 \mathrm{~nm}, 2 \mathrm{~nm}$ and $25 \mathrm{~nm}$, respectively. 


\section{WILEY-VCH}

\section{S4. Methods}

\section{Thin-Film Transistor Fabrication}

Bottom-gate, top-contact (BGTC) field-effect transistors were fabricated on highly doped $\left(\mathrm{n}^{++}\right)$silicon wafers, acting as the common gate electrode, with a $400 \mathrm{~nm}$ thermally grown silicon dioxide $\left(\mathrm{SiO}_{2}\right)$ layer as gate dielectric. The dielectric capacitance of the $\mathrm{SiO}_{2}$ layer was $8.6 \mathrm{nFcm}^{-2} . \mathrm{ZnO}$ films were spin-cast from the solutions described in The Experimental Section of the main text. The as-spun samples were annealed at $200{ }^{\circ} \mathrm{C}$ for 30 minutes in air. $50 \mathrm{~nm}$ aluminum source and drain electrodes were then deposited via thermal evaporation under high vacuum through shadow masks at an average rate of $0.5 \AA^{-1}$. Electrical characterization of all devices was carried out at room temperature under ambient pressure in $\mathrm{N}_{2}$, using an Agilent B2902A semiconductor parameter analyzer. The mobility of charge carriers was estimated in the linear and saturation regimes using the gradual-channel approximation. ${ }^{[6]}$

\section{References for SI}

[1] J. Tauc, R. Grigorovici, A. Vancu, physica status solidi (b) 1966, 15, 627; J. Tauc, Materials Research Bulletin 1968, 3, 37.

[2] H. Mizuta, T. Tanoue, The Physics and Applications of Resonant Tunnelling Diodes, Cambridge University Press, 2006.

[3] R. Tsu, L. Esaki, Appl. Phys. Lett. 1973, 22, 562.

[4] B. Enright, D. Fitzmaurice, The Journal of Physical Chemistry 1996, 100, 1027.

[5] W. S. Baer, Physical Review 1967, 154, 785.

[6] J. Zaumseil, H. Sirringhaus, Chemical Reviews 2007, 107, 1296. 